Purdue University

Purdue e-Pubs

Purdue CIBER Working Papers

Krannert Graduate School of Management

$1-1-2005$

\title{
Dynamic Learning, Pricing, and Ordering by a Censored Newsvendor
}

Arnab Bisi

Purdue University

Maqbool Dada

Purdue University

Follow this and additional works at: http:// docs.lib.purdue.edu/ciberwp

Bisi, Arnab and Dada, Maqbool, "Dynamic Learning, Pricing, and Ordering by a Censored Newsvendor " (2005). Purdue CIBER Working Papers. Paper 40.

http://docs.lib.purdue.edu/ciberwp/40

This document has been made available through Purdue e-Pubs, a service of the Purdue University Libraries. Please contact epubs@purdue.edu for additional information. 


\section{Dynamic Learning, Pricing, and Ordering by a Censored Newsvendor}

\section{Arnab Bisi and Maqbool Dada}

Purdue University

2005-005 


\title{
Dynamic Learning, Pricing, and Ordering by a Censored Newsvendor
}

\author{
Arnab Bisi Maqbool Dada \\ Krannert Graduate School of Management
}

Purdue University

West Lafayette, Indiana 47907-2056

abisi@mgmt.purdue.edu dada@mgmt.purdue.edu

Original Version: April 7, 2004

Revised Version

September 29, 2005 


\begin{abstract}
We address the problem of determining optimal ordering and pricing policies in a finite-horizon newsvendor model with unobservable lost sales. The demand distribution is price-dependent and involves unknown parameters. We consider both the cases of perishable and non-perishable inventory. A very general class of demand functions is studied in this paper. We derive the optimal ordering and pricing policies as unique functions of the stocking factor (which is a linear transformation of the safety factor). An important expression is obtained for the marginal expected value of information. As a consequence, we show when lost sales are unobservable, with perishable inventory the optimal stocking factor is always at least as large as the one given by the single-period model; whereas, if inventory is non-perishable, this result holds only under a strong condition. This expression also helps to explain why the optimal stocking factor of a period may not increase with the length of the problem. We compare this behavior with that of a full information model. We further examine the implications of the results to the special cases when demand uncertainty is described by additive and multiplicative models. For the additive case, we show that if demand is censored, the optimal policy is to order more as well as charge higher retail prices when compared to the policies in the single-period model and the full information model. We also compare optimal and myopic policies for the additive and multiplicative models.
\end{abstract}

Key words: Inventory. Bayesian Markov decision processes. Unknown demand. Lost sales. Censoring. Optimal policies. Myopic policies. 
Recent procurement strategies such as Quick Response that are supported by innovations in supply chain management practice provide opportunities for a retailer to order frequently over a product's life cycle. The option to place multiple orders not only allows the retailer to order a smaller order quantity initially, but also it allows the retailer to use the history of sales to suitably revise and refine the demand forecast so that orders more precisely mirror the retailer's current knowledge of the uncertainty in demand. In addition, the retailer can proactively adjust order quantities to enhance the rate of learning. Such ordering decisions can influence and are influenced by the pricing strategy of the retailer.

It is the goal of this paper to characterize the optimal dynamic ordering and pricing policies of a retailer whose uncertain demand is price-dependent with a very general functional form, and who has the opportunity to learn from its history of sales. In particular, we consider an $N$-period discrete time model in which at the start of each period, the retailer must determine the order quantity and price before observing the random demand for that period. If demand is less than available inventory, all demand is met. Demand in excess of available inventory is lost and unobserved. The demand observation is therefore censored by the inventory level and the retailer records the sales but not the demand in each period. In $\S 2$, we consider models in which leftover inventory is perishable, while the case of non-perishable inventory is considered in $\S 3$. We explicitly assume that while the distribution of demand is known some of its parameters are perceived as random variables with their own prior distribution. Thus, the data allow our decision maker who is a newsvendor, since decisions are made before observing demand, to use the sales history (with some censored demand observations) to update the prior distribution using a Bayesian scheme.

The Bayesian approach has been used by Scarf (1959, 1960), Karlin (1960), Iglehart (1964), Murray and Silver (1966), Azoury (1985), and Lovejoy (1990) to study dynamic inventory problems with observable demand. Inventory policy updating for censored demand is addressed by Harpaz, Lee and Winkler (1982), Braden and Freimer (1991), Nahmias (1994), Agrawal and Smith (1996), Lariviere and Porteus (1999), and Ding, Puterman and Bisi (2002). Adaptive inventory control for partially observed 
systems has been studied by Lovejoy (1993), Treharne and Sox (2002), and the references thereof. All these works assume that the retail price is fixed and set exogenously.

While the literature on joint stocking and pricing without demand learning is very extensive (see, for example, the paper by Petruzzi and Dada 1999), the literature on simultaneous stocking and pricing in the censored newsvendor model is scarce; representative papers include mainly those of Petruzzi and Dada (2001, 2002). In the stream of existing literature, the perishable inventory version of our model may be viewed as adding the pricing decisions to the problem considered by Ding, Puterman and Bisi (2002). We adapt their Markovian decision process framework to show that the problem representation can be reduced to determining the optimal values of $z_{n}$, the so called stocking factors. As explained by Petruzzi and Dada (1999), in a single-period newsvendor setting, the stocking factor in each period is a standardized transformation of the safety stock (the order quantity less the expected demand).

This transformation is then used to derive important identities for the marginal expected value of information for the perishable inventory case (Theorem 1), and marginal expected value of leftover inventory and information for the non-perishable inventory case (Theorem 2). These identities are then used to explain the counter-intuitive result that $z_{1}$ (also $z_{n}$ in general) need not be non-decreasing in $N$, the length of the problem; the latter result was first reported by Petruzzi and Dada (2002). In particular, we explain that the nested property holds in the model of Petruzzi and Dada (2002) because their distribution of demand, given the unknown parameter is degenerate (deterministic). We further show that for a model with full information on demand, the optimal stocking factors are always equal for different problem lengths when inventory is perishable; whereas, for the case of non-perishable inventory, this result is true only under a strong condition. Analogous results hold also for the observable lost sales models with either known or unknown demand distribution as these are special cases of our full information model.

For both the perishable inventory and the non-perishable cases we also show that 1) $z_{n} \geq z_{n}^{F}$, the stocking factor when demand rather than sales are observed. This extends to a generalized demand 
model with $N$ periods, a result previously proven by Dada and Petruzzi (2001) for an additive demand model with two periods. And, we also show that 2) as in Ding, Puterman and Bisi (2002), for the perishable inventory case, $z_{n} \geq z_{n}^{B N}$, the myopic stocking factor which is a solution to the one period problem faced by the newsvendor with the same information set. We further show that 3) for the case of non-perishable inventory, $z_{n} \geq z_{n}^{B N}$ holds only under a restrictive condition on the order placement.

We also derive the optimal ordering and pricing policies and their properties for additive and multiplicative models which are special cases of our general demand function. For the additive case, we show that the optimal policy in the presence of censored demand is to order more from the supplier as well as charge higher retail prices to the consumers than in either the single-period model and the full information model. We find that the optimal policies in the additive model have identical structures for both the cases of perishable and non-perishable inventory, but the structures are different in the case of the multiplicative model. Finally, we draw comparisons between the optimal and myopic policies for the additive and multiplicative demands.

As can be readily seen from the discussion above, one contribution of our paper is that it provides a unified modeling structure by providing a formulation of the censored newsvendor problem with a general price-dependent demand function that includes most of the limited number of published papers on the censored newsvendor problem. In addition to structural results, we also make some technical contributions since we are able to show that 1) the likelihood-ratio ordering assumption on the demand distribution is not required in Ding, Puterman and Bisi (2002); and, 2) one of our results not only helps to correct a condition in Petruzzi and Dada (2001) but also shows that their condition is not required.

The paper is organized as follows. In $\S 1$ we formulate the dynamic program for the censored newsvendor problem. Various properties of the optimal policies for perishable and non-perishable products are established in $\S \S 2$ and 3 respectively. In $\S 4$ we focus on additive and multiplicative demand models. $\S 5$ concludes the paper. Proofs of all the results are presented in the Appendix. 


\section{The Bayesian Censored Newsvendor Problem}

We formulate a newsvendor model for a single product whose demand distribution is price-dependent and involves unknown parameter(s). The objective of the newsvendor is to determine the joint pricing and ordering policies in each period over a finite-horizon of $N$ periods that maximize the total expected profit. After the pricing and ordering decisions are made at the beginning of each period, demand is realized. If demand is less than the order quantity, sales = demand, and there is leftover inventory. If demand exceeds the order quantity, sales = order quantity, and there is no leftover inventory. Demand in excess of the order quantity is lost and unobserved. The demand observation is therefore censored by the inventory level and the newsvendor observes the sales but not the demand in each period. Demand in period $n$ is modeled as (Young 1978)

$$
D_{n}\left(r_{n}, X_{n}\right)=d_{1}\left(r_{n}\right)+d_{2}\left(r_{n}\right) X_{n}, n=1,2, \ldots, N
$$

where $d_{1}\left(r_{n}\right)$ and $d_{2}\left(r_{n}\right)$ are decreasing functions of the per unit retail price $r_{n}, d_{2}\left(r_{n}\right)>0$, and $X_{n}$ is a random variable that captures the uncertainty in the demand function. Equation (1) represents a broad class of demands, including the case of exogenously set price. As particular examples we will consider $d_{1}\left(r_{n}\right)=a-b r_{n}(a>0, b>0)$ and $d_{2}\left(r_{n}\right)=1$, to model additive demand, and $d_{2}\left(r_{n}\right)=a r_{n}^{-b}(a>$ $0, b>1)$ and $d_{1}\left(r_{n}\right)=0$, to model multiplicative demand. We assume that the random variables $X_{n}, n=1,2, \ldots, N$, in different periods are independent and identically distributed (iid). In each period, $X_{n}$ is generated by a probability distribution with known density $f(\cdot \mid \theta)$ and unknown parameter (or

vector of parameters) $\boldsymbol{\theta}$ with realization $\theta \in \Theta$. In order to develop a common analytical procedure for the general model represented by Equation (1) which captures both additive and multiplicative demands as specific cases, we assume throughout this paper that $X_{n}$ is non-negative. The analysis can be easily modified to allow negative values of $X_{n}$ that may arise in some additive demand models. The costs for the newsvendor model in each period are a variable ordering cost of $c$ per unit and a penalty $p$ per unit short. If the inventory is perishable, the newsvendor gets a salvage value of $h$ per unit surplus in each 
period. Otherwise, for non-perishable inventory, the newsvendor incurs a holding cost of $h^{\prime}$ per unit surplus at the end of each period. It is reasonable to assume $h<c<p$ when inventory is perishable and $c<p$ when inventory is non-perishable. In this section as well as the next section, we consider the case of perishable products, whereas the non-perishable case is addressed in $\S 3$.

It is convenient to define

$$
z_{n}=\frac{y_{n}-d_{1}\left(r_{n}\right)}{d_{2}\left(r_{n}\right)}
$$

where $y_{n}$ denotes the stock level chosen in period $n$. The variable $z_{n}$ is called the stocking factor in period $n$ (Petruzzi and Dada 1999). Let $S F_{n} \equiv \frac{y_{n}-E\left[D_{n}\left(r_{n}, X_{n}\right)\right]}{S D\left[D_{n}\left(r_{n}, X_{n}\right)\right]}$ be the safety factor (Silver, Pyke and Peterson 1998), where SD represents the standard deviation. The stocking factor and the safety factor are related as follows: $z_{n}=E\left[X_{n}\right]+\left(S F_{n}\right)\left(S D\left[X_{n}\right]\right)$. The transformation (2) allows us to accommodate time dependent shifts in the expected demand that may arise for seasonal products, or for products that are in the early stages of their life cycles.

Let us denote $x_{n}=\min \left(X_{n}, z_{n}\right)$. Demand is exactly observed in period $n$, that is, $D_{n}\left(r_{n}, X_{n}\right)<y_{n}$ when $x_{n}<z_{n}$; and the demand is censored at the order quantity, that is, $D_{n}\left(r_{n}, X_{n}\right) \geq y_{n}$ when $x_{n}=z_{n}$. By comparing the sales and the order quantities, we can conclude if the demand equals sales or is at least as great as sales.

We now formulate the price-dependent newsvendor problem as a Bayesian Markov decision process (van Hee 1978, Ding, Puterman and Bisi 2002) as follows:

Decision Epochs. Let $N$ denote the finite number of decision epochs.

States. Let $S_{n}$ denote the state space at decision epoch $n, n=1,2, \ldots, N$. The state space is the set of all prior distributions when $n=1$ and posterior distributions when $n=2, \ldots, N$. We assume a fixed prior distribution $\pi_{1}(\theta)$. Then $S_{n}$ is the set of all posteriors corresponding to the given prior $\pi_{1}(\theta)$. Let $\hat{\pi}_{n+1}\left(\theta \mid x_{n}\right)$ be the posterior probability at decision epoch $n$. It is given by

$$
\hat{\pi}_{n+1}\left(\theta \mid x_{n}\right) \equiv \begin{cases}\pi_{n+1}\left(\theta \mid x_{n}\right) & \text { if } x_{n}<z_{n} \\ \pi_{n+1}^{c}\left(\theta \mid z_{n}\right) & \text { if } x_{n}=z_{n}\end{cases}
$$


The superscript $c$ represents censored demand. When the demand in period $n$ is fully observed, that is, when $x_{n}<z_{n}$, then if the prior is $\hat{\pi}_{n}(\theta)$, the state at $n+1$ becomes $\hat{\pi}_{n+1}\left(\theta \mid x_{n}\right)=\pi_{n+1}\left(\theta \mid x_{n}\right)$ as given by

$$
\pi_{n+1}\left(\theta \mid x_{n}\right)=\frac{f\left(x_{n} \mid \theta\right) \hat{\pi}_{n}(\theta)}{\int_{\Theta} f\left(x_{n} \mid \theta^{\prime}\right) \hat{\pi}_{n}\left(\theta^{\prime}\right) d \theta^{\prime}} .
$$

On the other hand, if the demand is censored at the order quantity, that is, when $x_{n}=z_{n}$, the state becomes $\hat{\pi}_{n+1}\left(\theta \mid x_{n}\right)=\pi_{n+1}^{c}\left(\theta \mid z_{n}\right)$, which is given by

$$
\pi_{n+1}^{c}\left(\theta \mid z_{n}\right)=\frac{\int_{z_{n}}^{\infty} f(x \mid \theta) d x \hat{\pi}_{n}(\theta)}{\int_{\Theta} \int_{z_{n}}^{\infty} f\left(x \mid \theta^{\prime}\right) \hat{\pi}_{n}\left(\theta^{\prime}\right) d x d \theta^{\prime}} .
$$

Note that the above sequence of prior and posterior probabilities constitute a distribution-valued Markov process which is the basis for dynamic demand distribution updating.

Actions. Since any non-negative stock level (or stocking factor) and price can be chosen at any decision epoch, the action set is $A_{s}=[0, \infty) \times[0, \infty)$ for each $s \in S_{n}$.

Expected Profit. Let us denote $\hat{g}_{n}(x)=\int_{\Theta} f(x \mid \theta) \hat{\pi}_{n}\left(\theta \mid x_{n-1}\right) d \theta$, the Bayesian estimate of the updated probability density function of $X_{n}$ conditioned on $X_{n-1}$, and $\hat{G}_{n}(x)=\operatorname{Prob}\left(X_{n} \leq x\right)=\int_{0}^{x} \hat{g}_{n}(s) d s$. Depending on whether $\hat{\pi}_{n}$ is evaluated based on an exact demand observation or a censored observation as indicated in Equation (3), the density $\hat{g}_{n}$ consists of the corresponding two cases as follows:

$$
\hat{g}_{n}(x) \equiv \begin{cases}g_{n}(x) & \text { if } \hat{\pi}_{n}\left(\theta \mid x_{n-1}\right)=\pi_{n}\left(\theta \mid x_{n-1}\right), \text { i.e., when } x_{n-1}<z_{n-1} \\ g_{n}^{c}(x) & \text { if } \hat{\pi}_{n}\left(\theta \mid x_{n-1}\right)=\pi_{n}^{c}\left(\theta \mid z_{n-1}\right), \text { i.e., when } x_{n-1}=z_{n-1} .\end{cases}
$$

Now, the Bayesian expected profit with prior distribution $\hat{\pi}_{n}$, stocking factor $z_{n}$, stock level $y_{n}$, and price $r_{n}$ can be written as

$$
M\left(\hat{\pi}_{n}, z_{n}, y_{n}, r_{n}\right)=\left(r_{n}-c\right) y_{n}-d_{2}\left(r_{n}\right)\left[\left(r_{n}-h\right) \int_{0}^{z_{n}} \hat{G}_{n}(x) d x+p \int_{z_{n}}^{\infty}\left(x-z_{n}\right) \hat{g}_{n}(x) d x\right] .
$$

After substituting $z_{n}$ for $y_{n}$ from (2) and performing integration by parts we write $M\left(\hat{\pi}_{n}, z_{n}, y_{n}, r_{n}\right)$ as $M\left(\hat{\pi}_{n}, z_{n}, r_{n}\right)$ by

$$
\begin{aligned}
M\left(\hat{\pi}_{n}, z_{n}, r_{n}\right)= & d_{1}\left(r_{n}\right)\left(r_{n}-c\right)+d_{2}\left(r_{n}\right)\left[\left(r_{n}+p-c\right) z_{n}-\left(r_{n}+p-h\right) z_{n} \hat{G}_{n}\left(z_{n}\right)\right] \\
& +d_{2}\left(r_{n}\right)\left[\left(r_{n}-h\right) \int_{0}^{z_{n}} x \hat{g}_{n}(x) d x-p \int_{z_{n}}^{\infty} x \hat{g}_{n}(x) d x\right] .
\end{aligned}
$$




\section{Transition Probabilities.}

$$
p\left(\hat{\pi}_{n+1} \mid \hat{\pi}_{n}, z_{n}, r_{n}\right)= \begin{cases}\hat{g}_{n}\left(x_{n}\right) d x_{n} & \text { if } \hat{\pi}_{n+1}\left(\theta \mid x_{n}\right)=\pi_{n+1}\left(\theta \mid x_{n}\right), \text { i.e., when } x_{n}<z_{n} \\ 1-\hat{G}_{n}\left(z_{n}\right) & \text { if } \hat{\pi}_{n+1}\left(\theta \mid x_{n}\right)=\pi_{n+1}^{c}\left(\theta \mid z_{n}\right), \text { i.e., when } x_{n}=z_{n} .\end{cases}
$$

The transition probability equals 0 otherwise. Note that the transition probabilities depend on the actions.

Optimality Equations. Under the total expected reward criterion, the optimality equations are given by

$$
\begin{aligned}
V_{n}\left(\hat{\pi}_{n}\right)= & \max _{z_{n}, r_{n} \in R_{+}}\left\{M\left(\hat{\pi}_{n}, z_{n}, r_{n}\right)+\int_{0}^{z_{n}} V_{n+1}\left(\pi_{n+1}(\cdot \mid x)\right) \hat{g}_{n}(x) d x\right. \\
& \left.+V_{n+1}\left(\pi_{n+1}^{c}\left(\cdot \mid z_{n}\right)\right)\left[1-\hat{G}_{n}\left(z_{n}\right)\right]\right\},
\end{aligned}
$$

for $n=1,2, \ldots, N$, with the boundary condition $V_{N+1}\left(\hat{\pi}_{N+1}\right)=0$, for all $\hat{\pi}_{N+1}$. The second term on the right hand side of Equation (9) is the expected profit at $n+1$ if $D_{n}\left(r_{n}, X_{n}\right)$ is fully observed (i.e. $\left.X_{n}<z_{n}\right)$ and the third term is the expected profit if $D_{n}\left(r_{n}, X_{n}\right)$ is censored at $y_{n}$ (i.e. $\left.X_{n} \geq z_{n}\right)$.

\section{The Perishable Inventory Problem}

In this section we establish important results of the optimal ordering and pricing policies for perishable products. For this, we first introduce some notation. For $n=1,2, \ldots, N$, let

$g_{n}\left(x \mid x_{n-1}\right)$ : the probability density of $X_{n}$ corresponding to the prior $\pi_{n}\left(\theta \mid x_{n-1}\right)$ which is the posterior when the demand in period $n-1$ is observed exactly (i.e., $\left.X_{n-1}=x_{n-1}\right)$.

$g_{n}^{c}\left(x \mid z_{n-1}\right)$ : the probability density of $X_{n}$ corresponding to the prior $\pi_{n}^{c}\left(\theta \mid z_{n-1}\right)$ which is the posterior when the demand in period $n-1$ is censored at the order quantity $y_{n-1}$ (i.e., $X_{n-1} \geq z_{n-1}$ ).

$G_{n}\left(x \mid x_{n-1}\right)=\operatorname{Prob}\left(X_{n} \leq x \mid X_{n-1}=x_{n-1}\right), \quad G_{n}^{c}\left(x \mid z_{n-1}\right)=\operatorname{Prob}\left(X_{n} \leq x \mid X_{n-1} \geq z_{n-1}\right)$. $z_{n}^{* e}$ : optimal stocking factor in period $n$ with state $\pi_{n}\left(\theta \mid x_{n-1}\right)$. 
$z_{n}^{* c}$ : optimal stocking factor in period $n$ with state $\pi_{n}^{c}\left(\theta \mid z_{n-1}\right)$.

$z_{n}^{e}=\left[G_{n}\left(\frac{r_{n}\left(z_{n}^{e}\right)+p-c}{r_{n}\left(z_{n}^{e}\right)+p-h} \mid x_{n-1}\right)\right]^{-1}:$ Bayesian myopic stocking factor in period $n$ if $X_{n-1}=x_{n-1}$.

$z_{n}^{c}=\left[G_{n}^{c}\left(\frac{r_{n}\left(z_{n}^{c}\right)+p-c}{r_{n}\left(z_{n}^{c}\right)+p-h} \mid z_{n-1}\right)\right]^{-1}:$ Bayesian myopic stocking factor in period $n$ if $X_{n-1} \geq z_{n-1}$.

Note that by the Bayesian myopic stocking factor we refer to the stocking factor that corresponds to the optimal solution of the single-period problem with the updated probability distribution.

We denote the optimal and myopic stocking factors by

$$
\begin{gathered}
z_{n}^{*}= \begin{cases}z_{n}^{* e} & \text { if } X_{n-1}=x_{n-1}, \text { i.e., when } x_{n-1}<z_{n-1} \\
z_{n}^{* c} & \text { if } X_{n-1} \geq z_{n-1}, \text { i.e., when } x_{n-1}=z_{n-1} .\end{cases} \\
z_{n}^{B N}= \begin{cases}z_{n}^{e} & \text { if } X_{n-1}=x_{n-1}, \text { i.e., when } x_{n-1}<z_{n-1} \\
z_{n}^{c} & \text { if } X_{n-1} \geq z_{n-1}, \text { i.e., when } x_{n-1}=z_{n-1} .\end{cases}
\end{gathered}
$$

We will write $z_{n}^{*} \geq z_{n}^{B N}$ (or $z_{n}^{*}=z_{n}^{B N}$ ) to mean that $z_{n}^{* e} \geq z_{n}^{e}$ if $X_{n-1}=x_{n-1}<z_{n-1}$, and $z_{n}^{* c} \geq z_{n}^{c}$ if $X_{n-1} \geq z_{n-1}=x_{n-1}\left(\right.$ or $z_{n}^{* e}=z_{n}^{e}$ if $X_{n-1}=x_{n-1}<z_{n-1}$, and $z_{n}^{* c}=z_{n}^{c}$ if $\left.X_{n-1} \geq z_{n-1}=x_{n-1}\right)$.

In Equation (3) we have seen how $\hat{\pi}_{n}\left(\cdot \mid x_{n-1}\right), n=2,3, \ldots, N$, are calculated depend on whether $D_{n-1}\left(r_{n-1}, X_{n-1}\right)$ is fully observed or censored. Hence,

$$
\equiv \begin{array}{ll}
V_{n}\left(\hat{\pi}_{n}\left(\cdot \mid x_{n-1}\right)\right) \\
\equiv \begin{array}{ll}
V_{n}\left(\pi_{n}\left(\cdot \mid x_{n-1}\right)\right) & \text { if } \hat{\pi}_{n}\left(\cdot \mid x_{n-1}\right)=\pi_{n}\left(\cdot \mid x_{n-1}\right), \text { i.e., when } X_{n-1}=x_{n-1}<z_{n-1} \\
V_{n}\left(\pi_{n}^{c}\left(\cdot \mid z_{n-1}\right)\right) & \text { if } \hat{\pi}_{n}\left(\cdot \mid x_{n-1}\right)=\pi_{n}^{c}\left(\cdot \mid z_{n-1}\right), \text { i.e., when } X_{n-1} \geq x_{n-1}=z_{n-1} .
\end{array}
\end{array}
$$

Since $\pi_{n}\left(\cdot \mid x_{n-1}\right)$ can be viewed as a function of $\hat{\pi}_{n-1}$ and $x_{n-1}$ (refer to Equation (4)), and $\pi_{n}^{c}\left(\cdot \mid z_{n-1}\right)$ can be viewed as a function of $\hat{\pi}_{n-1}$ and $z_{n-1}$ (refer to Equation (5)), we redefine $V_{n}\left(\hat{\pi}_{n}\left(\cdot \mid x_{n-1}\right)\right)$ as

$$
V_{n}\left(\hat{\pi}_{n}\left(\cdot \mid x_{n-1}\right)\right) \equiv \begin{cases}V_{n}\left(\hat{\pi}_{n-1}, x_{n-1}\right) & \text { if } x_{n-1}<z_{n-1} \\ V_{n}^{c}\left(\hat{\pi}_{n-1}, z_{n-1}\right) & \text { if } x_{n-1}=z_{n-1} .\end{cases}
$$

For notational convenience, we will denote

$$
V_{n}\left(\hat{\pi}_{n-1}, x_{n-1}\right) \equiv V_{n}\left(x_{n-1}\right), \quad V_{n}^{c}\left(\hat{\pi}_{n-1}, z_{n-1}\right) \equiv V_{n}^{c}\left(z_{n-1}\right)
$$

The following lemma specifies the optimal pricing and ordering decisions as functions of the stocking factor. In Example 1 of this section as well as in Lemmas 3 and 4 in $\S 4$ we will show how these equations admit simple solutions for the additive and multiplicative specifications of demand. 
Lemma 1. The optimal retail price and order quantity in period $n, n=1,2, \ldots, N$, are uniquely determined as functions of $z_{n}$ as follows:

(a) $r_{n}^{*}\left(z_{n}\right)$ is obtained from the equation:

$$
\begin{aligned}
& d_{1}\left(r_{n}\right)+d_{2}\left(r_{n}\right) z_{n}+\left(r_{n}-c\right)\left(\frac{\partial d_{1}\left(r_{n}\right)}{\partial r_{n}}+\frac{\partial d_{2}\left(r_{n}\right)}{\partial r_{n}} z_{n}\right)-\frac{\partial d_{2}\left(r_{n}\right)}{\partial r_{n}}\left(r_{n}-h\right) \int_{0}^{z_{n}} \hat{G}_{n}(x) d x \\
& -d_{2}\left(r_{n}\right) \int_{0}^{z_{n}} \hat{G}_{n}(x) d x-\frac{\partial d_{2}\left(r_{n}\right)}{\partial r_{n}} p \int_{z_{n}}^{\infty}\left(x-z_{n}\right) \hat{g}_{n}(x) d x=0,
\end{aligned}
$$

(b) $y_{n}^{*}\left(z_{n}\right)=d_{1}\left(r_{n}^{*}\left(z_{n}\right)\right)+d_{2}\left(r_{n}^{*}\left(z_{n}\right)\right) z_{n}$.

Let us define the optimal future profit and the profit function at period $\mathrm{n}$ respectively by

$$
\begin{gathered}
I_{n}\left(z_{n}\right)=\int_{0}^{z_{n}} V_{n+1}(x) \hat{g}_{n}(x) d x+V_{n+1}^{c}\left(z_{n}\right)\left[1-\hat{G}_{n}\left(z_{n}\right)\right] \\
J\left(\hat{\pi}_{n}, z_{n}\right)=M\left(\hat{\pi}_{n}, z_{n}, r_{n}\left(z_{n}\right)\right)+I_{n}\left(z_{n}\right) .
\end{gathered}
$$

Then, in view of Lemma 1(a), the optimality equations (9) can be written as

$$
V_{n}\left(\hat{\pi}_{n}\right)=\max _{z_{n} \in R_{+}} J\left(\hat{\pi}_{n}, z_{n}\right)
$$

for $n=1,2, \ldots, N$, with the boundary condition $V_{N+1}\left(\hat{\pi}_{N+1}\right)=0$ for all $\hat{\pi}_{N+1}$. Notice that in Equation (16), the optimization problem of jointly ordering and pricing has been reduced to a single-variable problem in the stocking factor $z_{n}$.

We now derive an important result for the marginal expected value of information. Arguing similarly to Ding, Puterman, and Bisi (2002) we can write the marginal expected value of information at $z_{n}$ as

$$
\begin{aligned}
i_{n}\left(z_{n}\right) & =\frac{d V_{n+1}^{c}\left(z_{n}\right)}{d z_{n}}-\left(V_{n+1}^{c}\left(z_{n}\right)-V_{n+1}\left(z_{n}\right)\right) \frac{\hat{g}_{n}\left(z_{n}\right)}{1-\hat{G}_{n}\left(z_{n}\right)} \\
& \equiv \frac{1}{1-\hat{G}_{n}\left(z_{n}\right)} \frac{d I_{n}\left(z_{n}\right)}{d z_{n}}, \quad \text { for } n=1,2, \ldots, N-1
\end{aligned}
$$

By appropriately analyzing Equation (17), Theorem 1(a) establishes a more pragmatic expression for $i_{n}\left(z_{n}\right)$. Theorem $1(\mathrm{~b})$ states how this expression can be used to compute the optimal stocking factor. 
We use this expression later in this section to explain why the optimal stocking factor of a period may not increase with the length of the problem horizon.

Theorem 1. (a) The marginal expected value of information $i_{n}\left(z_{n}\right)$ can be expressed as

$$
i_{n}\left(z_{n}\right)=\hat{h}_{n}\left(z_{n}\right)\left(V_{n+1}\left(z_{n}\right)-\left.V_{n+1}\left(z_{n}\right)\right|_{z_{n+1}=z_{n+1}^{* c}}\right), n=1,2, \ldots, N-1
$$

where $\hat{h}_{n}\left(z_{n}\right)=\frac{\hat{g}_{n}\left(z_{n}\right)}{1-\hat{G}_{n}\left(z_{n}\right)}$ and $\left.V_{n+1}\left(z_{n}\right)\right|_{z_{n+1}=z_{n+1}^{* c}}$ is the profit when $V_{n+1}\left(z_{n}\right)$ is evaluated at $z_{n+1}=z_{n+1}^{* c}$ in period $(n+1)$, and subsequently at $z_{n+j}=z_{n+j}^{*}$ in period $(n+j), j=2,3, \ldots, N-n$, given that the policy used in period $(n+1)$ is $z_{n+1}^{* c} .{ }^{1}$

(b) The optimal stocking factor $z_{n}^{*}$ satisfies

$$
d_{2}\left(r_{n}\left(z_{n}\right)\right)(c-h) \hat{G}_{n}\left(z_{n}\right)=\left[1-\hat{G}_{n}\left(z_{n}\right)\right]\left[d_{2}\left(r_{n}\left(z_{n}\right)\right)\left(r_{n}\left(z_{n}\right)+p-c\right)+i_{n}\left(z_{n}\right)\right]
$$

for $n=1,2, \ldots, N-1$.

Explicit expressions for $V_{n+1}\left(z_{n}\right)$ and $\left.V_{n+1}\left(z_{n}\right)\right|_{z_{n+1}=z_{n+1}^{* c}}$ are given in Equations (42) and (64) respectively of the Appendix. Equation (18) can be interpreted as follows. Given that the demand is censored in period $n$, that is, $X_{n} \geq z_{n}$, suppose we have the option of acquiring additional information by purchasing additional inventory that is sufficient to increase the stocking factor by $d z_{n}$. If this additional inventory turns out to be an excess, then the optimal policy in period $(n+1)$ is $z_{n+1}^{* e}$ and the corresponding (optimal) profit is $V_{n+1}\left(z_{n}\right)$. On the other hand, if there is no excess inventory, then the optimal policy in period $(n+1)$ is $z_{n+1}^{* c}$ and the corresponding profit is $\left.V_{n+1}\left(z_{n}\right)\right|_{z_{n+1}=z_{n+1}^{* c}}$. The probability density function of $X_{n}$ at $z_{n}$ given $X_{n} \geq z_{n}$ is $\hat{h}_{n}\left(z_{n}\right)=\frac{\hat{g}_{n}\left(z_{n}\right)}{1-\hat{G}_{n}\left(z_{n}\right)}$. Therefore, the value of information at $z_{n}$ is the net increase in the future profit given by $\hat{h}_{n}\left(z_{n}\right)\left(V_{n+1}\left(z_{n}\right)-\left.V_{n+1}\left(z_{n}\right)\right|_{z_{n+1}=z_{n+1}^{* c}}\right)$, which is Equation (18). Now, since $V_{n+1}\left(z_{n}\right) \geq\left. V_{n+1}\left(z_{n}\right)\right|_{z_{n+1}=z_{n+1}^{* c}}$, Theorem 1(a) implies that the marginal expected value of information at any $z_{n}$ is always non-negative. For inventory models with exogenous price, a similar representation but with a different proof of Equation (18) has also been found independently by Lu, Song, and Zhu (2004).

\footnotetext{
${ }^{1}$ The correction in this result from the earlier draft is due to an anonymous referee.
} 
In the following example, we apply Lemma 1 and Theorem 1 to a specific problem to derive the equations that would give us the optimal policies.

Example 1. Let $X_{n}, n=1,2, \ldots, N$, be exponential random variables with distribution function $F(x \mid \theta)=1-e^{-\theta x}$, where $\boldsymbol{\theta}$ has the Gamma prior $\pi_{1}(\theta)=\frac{\beta^{\alpha}}{\Gamma(\alpha)} \theta^{\alpha-1} e^{-\beta \theta}$, with the shape and scale parameters, $\alpha$ and $\beta$, respectively. Noting that $x_{n}=\min \left(X_{n}, z_{n}\right)$, the sufficient statistic for $\theta$ at the beginning of period $n$ is given by the updated parameters $\alpha_{n}$ and $\beta_{n}$ as follows (Braden and Freimer 1991):

$$
\alpha_{n}=\alpha+\sum_{i=1}^{n-1} I_{\left\{X_{i}<z_{i}\right\}} \quad \text { and } \quad \beta_{n}=\beta+\sum_{i=1}^{n-1} x_{i} .
$$

The updated distribution function of $X_{n}$ is then given by $\hat{G}_{n}(x)=1-\left(\frac{\beta_{n}}{\beta_{n}+x}\right)^{\alpha_{n}}$. Now, consider the additive demand model with $d_{1}\left(r_{n}\right)=a-b r_{n}(a>0, b>0)$ and $d_{2}\left(r_{n}\right)=1$. In this case, by Equations (18) and (19), the optimal stocking factor $z_{n}^{*}$ satisfies

$$
1-\hat{G}_{n}\left(z_{n}\right)=\frac{c-h}{\left(r_{n}\left(z_{n}\right)+p-h\right)+\frac{\alpha_{n}}{\beta_{n}+z_{n}}\left(V_{n+1}\left(z_{n}\right)-\left.V_{n+1}\left(z_{n}\right)\right|_{z_{n+1}=z_{n+1}^{* c}}\right)}
$$

where the optimal retail price and order quantity are given by Lemma 1 (also see Lemma 3 in $\S 3$ ) as follows:

$$
\begin{aligned}
& r_{n}\left(z_{n}\right)=\frac{1}{2 b}\left[a+b c+\frac{\beta_{n}}{\alpha_{n}-1}\left(1-\left(\frac{\beta_{n}}{\beta_{n}+z_{n}}\right)^{\alpha_{n}-1}\right)\right] \\
& y_{n}\left(z_{n}\right)=\frac{1}{2}\left[a-b c-\frac{\beta_{n}}{\alpha_{n}-1}\left(1-\left(\frac{\beta_{n}}{\beta_{n}+z_{n}}\right)^{\alpha_{n}-1}\right)\right]+z_{n}
\end{aligned}
$$

As a consequence of Theorem 1, we now obtain the following corollary describing a structural property of the optimal stocking factor for the general demand functions modeled by Equation (1).

Corollary 1. (a) Suppose $X_{n}, n=1,2, \ldots, N$, are non-negative iid random variables with density $f(x \mid \theta)$. Then $I_{n}\left(z_{n}\right)$ is non-decreasing in $z_{n}, n=1,2, \ldots, N-1$.

(b) Suppose each single-period problem has a unique optimal myopic stocking factor $z_{n}^{B N}$. Then the optimal stocking factor $z_{n}^{*}$ satisfies

$$
z_{n}^{*} \geq z_{n}^{B N}, n=1,2, \ldots, N-1, \quad \text { and } \quad z_{N}^{*}=z_{N}^{B N}
$$


Corollary 1 (b) states that in each of the periods $1,2, \ldots, N-1$, the combined effect of the unknown demand distribution and unobservable lost sales results in a higher optimal stocking factor than the Bayesian myopic stocking factor. This is a well known result in the context of inventory models with exogenous price (Lariviere and Porteus 1999, Ding, Puterman and Bisi 2002). Note that the assumption in Corollary 1(b) is needed because, when price is a decision variable, the single-period profit function may not be unimodal in $z_{n}$. In such cases, we would not be able to compare $z_{n}^{*}$ and $z_{n}^{B N}$. In Corollary 2 discussed below, we give examples of demand models for which each single-period problem has a unique optimal myopic stocking factor under a condition on the hazard rate function.

In view of Theorem 1 for the additive demand case and Theorem 2 for the multiplicative demand case in Petruzzi and Dada (1999), the following corollary gives a condition under which our Corollary 1(b) holds for continuous demand.

Corollary 2. Let $\hat{h}_{n}\left(z_{n}\right)=\frac{\hat{g}_{n}\left(z_{n}\right)}{1-\hat{G}_{n}\left(z_{n}\right)}$ be the hazard rate function associated with $X_{n}, n=1,2, \ldots, N$. Then Corollary 1(b) holds for the additive and multiplicative demand models if the following condition holds:

$$
\text { Condition } C_{1}: \quad \frac{d \hat{h}_{n}\left(z_{n}\right)}{d z_{n}}+2\left(\hat{h}_{n}\left(z_{n}\right)\right)^{2}>0
$$

If Condition $C_{1}$ is not satisfied or is hard to verify, an exhaustive search for the optimal $z_{n}^{B N}$ may be needed to validate Corollary 1(b), as is the case when demand is discrete.

Example 2. Consider the distribution $F(x \mid \theta)=1-e^{-\theta n(x)}$ of $X_{n}$, where $n(x)$ is convex and increasing in $x$. This distribution belongs to a subclass of Newsboy Distributions (Braden and Freimer 1991). If we let $\pi_{1}(\theta)=\operatorname{Gamma}(\alpha, \beta)$ with the shape parameter $\alpha>\frac{1}{2}$, then the above distribution paired with gamma satisfies Condition C (Theorem 4, Petruzzi and Dada 2001). Note that for $n(x)=x^{\lambda}$ with $\lambda \geq 1$, $F(x \mid \theta)$ is a Weibull distribution, and in particular, when $\lambda=1, F(x \mid \theta)$ is an exponential distribution. 
The following proposition establishes an important property of the updated probability distribution of $X_{n}, n=2,3, \ldots, N$. Let $\hat{G}_{n}^{U}\left(x \mid z_{n-1}\right)$ denote the updated marginal distribution function of $X_{n}$ (after unconditioning w.r.t. $\left.X_{n-1}\right)$ when the stocking factor in period $(n-1)$ is $z_{n-1}$. Then $\hat{G}_{n}^{U}\left(x \mid z_{n-1}\right)$ is given by

$$
\begin{aligned}
\hat{G}_{n}^{U}\left(x \mid z_{n-1}\right) & =P\left(X_{n} \leq x \mid z_{n-1}\right) \\
& =\int_{0}^{z_{n-1}} G_{n}\left(x \mid x_{n-1}\right) \hat{g}_{n-1}\left(x_{n-1}\right) d x_{n-1}+G_{n}^{c}\left(x \mid z_{n-1}\right)\left[1-\hat{G}_{n-1}\left(z_{n-1}\right)\right] .
\end{aligned}
$$

The proposition states that $\hat{G}_{n}^{U}\left(x \mid z_{n-1}\right)$ does not depend on $z_{n-1}$. We use this result in $\S 5$ to analyze a condition in Petruzzi and Dada (2001).

Proposition 1. The probability distribution function $\hat{G}_{n}^{U}\left(x \mid z_{n-1}\right)$ satisfies

$$
\frac{\partial \hat{G}_{n}^{U}\left(x \mid z_{n-1}\right)}{\partial z_{n-1}}=0
$$

for $n=2,3, \ldots, N$.

We now examine a nested property of the optimal stocking factor. Let $z_{n, N}^{*}$ denote the optimal value of the stocking factor $z$ in period $n$ of an $N$-period problem. Petruzzi and Dada (2002) have proved that the first-period optimal stocking factor increases as $N$ increases, that is, the inequality $z_{1, N+1}^{*} \geq z_{1, N}^{*}$ holds for any $N$ in their analytical model. This phenomenon is also noted in a numerical example (Example 3, p 525) in Ding, Puterman and Bisi (2002). However, this property may not hold in general; neither for the first-period nor for any intermediate period $n$, where $n<N$. This behavior is highly counter-intuitive, because usually we would believe that acquiring demand information by stocking higher in the early periods is more valuable in an $(N+1)$-period problem than it is in an $N$-period problem. The explanation of this surprising feature of the stocking factor is given below.

Let $J_{N}\left(\hat{\pi}_{n}, z_{n}\right)$ and $V_{n, N}\left(\hat{\pi}_{n}\right)$ denote the profit function and the optimal profit (refer to Equations (15) and (16)) respectively in period $n$ of an $N$-period problem. Also let $I_{n, N}\left(z_{n}\right)$ denote the optimal future profit (refer to Equation (14)) from period $(n+1)$ onwards of an $N$-period problem given that $z_{n}$ 
is the decision in period $n$. Then, for a two-period problem, using Corollary 1(a), we get

$$
\begin{aligned}
\frac{d J_{2}\left(\pi_{1}, z_{1}\right)}{d z_{1}} & =d_{2}\left(r_{1}\left(z_{1}\right)\right)\left[\left(r_{1}\left(z_{1}\right)+p-c\right)-\left(r_{1}\left(z_{1}\right)+p-h\right) G_{1}\left(z_{1}\right)\right]+\frac{d I_{1,2}\left(z_{1}\right)}{d z_{1}} \\
& \geq d_{2}\left(r_{1}\left(z_{1}\right)\right)\left[\left(r_{1}\left(z_{1}\right)+p-c\right)-\left(r_{1}\left(z_{1}\right)+p-h\right) G_{1}\left(z_{1}\right)\right] \\
& =\frac{d J_{1}\left(\pi_{1}, z_{1}\right)}{d z_{1}}
\end{aligned}
$$

which implies

$$
z_{1,2}^{*} \geq z_{1,1}^{*}
$$

But neither the inequality $z_{1, N+1}^{*} \geq z_{1, N}^{*}$ nor the inequality $z_{n, N+1}^{*} \geq z_{n, N}^{*}$, for any $n<N$, is necessarily true for a problem with more than two periods. To show this, let us note the following equation:

$$
\frac{d J_{N+1}\left(\hat{\pi}_{n}, z_{n}\right)}{d z_{n}}=d_{2}\left(r_{n}\left(z_{n}\right)\right)\left[\left(r_{n}\left(z_{n}\right)+p-c\right)-\left(r_{n}\left(z_{n}\right)+p-h\right) \hat{G}_{n}\left(z_{n}\right)\right]+\frac{d I_{n, N+1}\left(z_{n}\right)}{d z_{n}} .
$$

Comparing $\frac{d J_{N+1}\left(\hat{\pi}_{n}, z_{n}\right)}{d z_{n}}$ and $\frac{d J_{N}\left(\hat{\pi}_{n}, z_{n}\right)}{d z_{n}}$ we observe that for $z_{n, N+1}^{*} \geq z_{n, N}^{*}$ to hold we must have $\frac{d I_{n, N+1}\left(z_{n}\right)}{d z_{n}} \geq \frac{d I_{n, N}\left(z_{n}\right)}{d z_{n}}$. Notice that by Theorem 1(a) and Equation (17) we can write

$$
\begin{aligned}
\frac{d I_{n, N+1}\left(z_{n}\right)}{d z_{n}}-\frac{d I_{n, N}\left(z_{n}\right)}{d z_{n}}= & \hat{g}_{n}\left(z_{n}\right)\left(V_{n+1, N+1}\left(z_{n}\right)-\left.V_{n+1, N+1}\left(z_{n}\right)\right|_{z_{n+1}=z_{n+1, N+1}^{* c}}\right) \\
& -\hat{g}_{n}\left(z_{n}\right)\left(V_{n+1, N}\left(z_{n}\right)-\left.V_{n+1, N}\left(z_{n}\right)\right|_{z_{n+1}=z_{n+1, N}^{* c}}\right) \\
= & \hat{g}_{n}\left(z_{n}\right)\left(V_{n+1, N+1}\left(z_{n}\right)-V_{n+1, N}\left(z_{n}\right)\right) \\
& -\hat{g}_{n}\left(z_{n}\right)\left(\left.V_{n+1, N+1}\left(z_{n}\right)\right|_{z_{n+1}=z_{n+1, N+1}^{* c}}-\left.V_{n+1, N}\left(z_{n}\right)\right|_{z_{n+1}=z_{n+1, N}^{* c}}\right) .
\end{aligned}
$$

The net effect of the two terms inside the first parenthesis on the right hand side of Equation (25), although non-negative, can be arbitrarily small. The net effect of the two terms inside the second parenthesis can be either positive or negative of arbitrary quantity, depending on the values of $z_{n+1, N+1}^{* c}$ and $z_{n+1, N}^{* c}$. Therefore, the inequality $\frac{d I_{n, N+1}\left(z_{n}\right)}{d z_{n}} \geq \frac{d I_{n, N}\left(z_{n}\right)}{d z_{n}}$ and hence, $z_{n, N+1}^{*} \geq z_{n, N}^{*}$ may be violated. An example of this case is presented graphically in Figure 1 where it is shown how the difference $\left(\left.V_{n+1, N+1}\left(z_{n}\right)\right|_{z_{n+1}=z_{n+1, N+1}^{* c}}-\left.V_{n+1, N}\left(z_{n}\right)\right|_{z_{n+1}=z_{n+1, N}^{* c}}\right)$ can be greater than $\left(V_{n+1, N+1}\left(z_{n}\right)-V_{n+1, N}\left(z_{n}\right)\right)$ $\equiv\left(\left.V_{n+1, N+1}\left(z_{n}\right)\right|_{z_{n+1}=z_{n+1, N+1}^{* e}}-\left.V_{n+1, N}\left(z_{n}\right)\right|_{z_{n+1}=z_{n+1, N}^{* e}}\right)$. The intuition behind why $z_{n, N+1}^{*} \geq z_{n, N}^{*}$ 
may not hold can be explained by the marginal expected value of information. From Equations (14)(16) note that

$$
\left.V_{n+1, N+1}\left(z_{n}\right)\right|_{z_{n+1}=z_{n+1, N+1}^{* c}}=M\left(\pi_{n+1}, z_{n+1, N+1}^{* c}, r_{n+1}\left(z_{n+1, N+1}^{* c}\right)\right)+I_{n+1, N+1}\left(z_{n+1, N+1}^{* c}\right),
$$

where

$$
\begin{aligned}
I_{n+1, N+1}\left(z_{n+1, N+1}^{* c}\right)= & \int_{0}^{z_{n+1, N+1}^{* c}} V_{n+2, N+1}(x) g_{n+1}(x) d x \\
& +V_{n+2, N+1}^{c}\left(z_{n+1, N+1}^{* c}\right)\left[1-G_{n+1}\left(z_{n+1, N+1}^{* c}\right)\right] .
\end{aligned}
$$

This indicates that the marginal expected value of information in period $n$ (refer to Equations (17) and (18) ) depends on the future profit from period $(n+1)$ onwards; in particular, on the profit functions $V_{n+2, N+1}(x)$ and $V_{n+2, N+1}^{c}\left(z_{n+1, N+1}^{* c}\right)$. By Corollary 1 (a) we observe that $I_{n+1, N+1}\left(z_{n+1, N+1}^{* c}\right) \geq$ $I_{n+1, N+1}\left(z_{n+1, N}^{* c}\right) \geq I_{n+1, N}\left(z_{n+1, N}^{* c}\right)$ if $z_{n+1, N+1}^{* c} \geq z_{n+1, N}^{* c}$. In such a case, observe from Equation (25) that the value of the terms in the second parenthesis can exceed that of the first one. Therefore, we may have $z_{n, N+1}^{*} \leq z_{n, N}^{*}$, albeit depending on the value of the difference between $M\left(\pi_{n+1}, z_{n+1, N+1}^{* c}, r_{n+1}\left(z_{n+1, N+1}^{* c}\right)\right)$ and $M\left(\pi_{n+1}, z_{n+1, N}^{* c}, r_{n+1}\left(z_{n+1, N}^{* c}\right)\right)$. An example where $z_{n+1, N+1}^{* c} \geq z_{n+1, N}^{* c}$ holds is given below. Consider a problem with $N=2$ and $n=1$. Then, by Equation (24) we have $z_{2,3}^{*} \geq z_{2,2}^{*}$, in particular, $z_{2,3}^{* c} \geq z_{2,2}^{* c}$, because $z_{2,3}^{*}$ can be thought of as the optimal stocking factor in the first period of a 2-period problem with $\hat{G}_{2}\left(x \mid z_{1}\right)$ as the initial distribution function of $X_{1}$.

The following insight can be obtained from the above discussion. If the number of period $N$ increases, the optimal stocking factor of a given period may not increase because there are now more periods available in the future when the optimal stocking factor has the opportunity to increase, if required, in order to gain information on the demand distribution. In contrast to the general model we analyze here, for the specific model of Petruzzi and Dada (2002), the first-period marginal expected value of information is independent of all future profits starting from the third-period profit functions $V_{3, N+1}(\cdot)$ and $V_{3, N+1}^{c}(\cdot)$. This happens as a consequence of the fact that in their model, the updated probability distribution is truncated at the lower bound of the uncertainty term. Such a construct also guarantees 
that having a higher stocking factor when a demand observation is censored makes the updated demand distribution stochastically larger with a higher precision parameter. The combination of these effects and the absence of any influence from future decisions leads to a higher optimal stocking factor in the first period as the problem length increases. But in our general model, not only is the stocking factor of a period influenced by future decisions but also the precision parameter is not guaranteed to increase with a higher stocking factor when demand is censored.

Note that above conclusion holds also for the specific inventory model of the exogenous-price case as in Ding, Puterman and Bisi (2002) as well as more general model of the endogenous-price case as in the multi-period version of Dana and Petruzzi (2001) where a firm internalizes the effect of its inventory on demand. Furthermore, the results in this section can be proved for both of these models.

\section{The Non-perishable Inventory Problem}

We now study the properties of the optimal policies when the inventory is non-perishable, that is, the leftover inventory from one period can be carried forward to the next period. Consequently, instead of a salvage value, the newsvendor incurs a holding cost of $h^{\prime}$ per unit of leftover inventories. Let $\xi_{n}$ and $q_{n}$ respectively denote the inventory level before ordering and the order quantity in period $n$. The stocking quantity in period $n$ is therefore $y_{n} \equiv \xi_{n}+q_{n}$. As before, the stocking factor $z_{n}$ is defined by Equation (2). The Bayesian MDP formulation of the problem is similar to the one in $\S 1$. To keep the discussion short, we only point out the changes that are necessary to analyze the results. For instance, Equation (8) is replaced by

$$
\begin{aligned}
M\left(\hat{\pi}_{n}, \xi_{n}, z_{n}, r_{n}\right)= & d_{1}\left(r_{n}\right)\left(r_{n}-c\right)+c \xi_{n}+d_{2}\left(r_{n}\right)\left[\left(r_{n}+p-c\right) z_{n}-\left(r_{n}+p+h^{\prime}\right) z_{n} \hat{G}_{n}\left(z_{n}\right)\right] \\
& +d_{2}\left(r_{n}\right)\left[\left(r_{n}+h^{\prime}\right) \int_{0}^{z_{n}} x \hat{g}_{n}(x) d x-p \int_{z_{n}}^{\infty} x \hat{g}_{n}(x) d x\right] .
\end{aligned}
$$


Also, the optimality equations (9) now takes the form

$$
\begin{aligned}
V_{n}\left(\hat{\pi}_{n}, \xi_{n}\right)= & \max _{z_{n} \in R_{+}}\left\{M\left(\hat{\pi}_{n}, \xi_{n}, z_{n}, r_{n}\left(z_{n}\right)\right)+\int_{0}^{z_{n}} V_{n+1}\left(\pi_{n+1}(\cdot \mid x), \xi_{n+1}\left(z_{n}\right)\right) \hat{g}_{n}(x) d x\right. \\
& \left.+V_{n+1}\left(\pi_{n+1}^{c}\left(\cdot \mid z_{n}\right), 0\right)\left[1-\hat{G}_{n}\left(z_{n}\right)\right]\right\},
\end{aligned}
$$

for $n=1,2, \ldots, N$, with the boundary condition $V_{N+1}\left(\hat{\pi}_{N+1}, \xi_{N+1}\right)=0$ for all $\hat{\pi}_{N+1}$ and $\xi_{N+1}$. Note that in Equation $(29), \xi_{n+1}\left(z_{n}\right) \equiv d_{2}\left(r_{n}\left(z_{n}\right)\right)\left(z_{n}-x\right)$ for $X_{n}=x<z_{n}$.

For notational simplicity, we denote

$$
V_{n+1}\left(\pi_{n+1}(\cdot \mid x), \xi_{n+1}\left(z_{n}\right)\right) \equiv V_{n+1}\left(x, \xi_{n+1}\left(z_{n}\right)\right), \quad V_{n+1}\left(\pi_{n+1}^{c}\left(\cdot \mid z_{n}\right), 0\right) \equiv V_{n+1}^{c}\left(z_{n}, 0\right)
$$

Analogous to Lemma 1, the optimal pricing and ordering policies for the non-perishable inventory model are given by the following lemma:

Lemma 2. The optimal price and order quantity can be expressed as unique functions of $z_{n}$ as follows:

(a) $\tilde{r}_{n}^{*}\left(z_{n}\right)$ is obtained by solving

$$
\begin{aligned}
& d_{1}\left(r_{n}\right)+d_{2}\left(r_{n}\right) z_{n}+\left(r_{n}-c\right)\left(\frac{\partial d_{1}\left(r_{n}\right)}{\partial r_{n}}+\frac{\partial d_{2}\left(r_{n}\right)}{\partial r_{n}} z_{n}\right)-\frac{\partial d_{2}\left(r_{n}\right)}{\partial r_{n}}\left(r_{n}+h^{\prime}\right) \int_{0}^{z_{n}} \hat{G}_{n}(x) d x \\
& -d_{2}\left(r_{n}\right) \int_{0}^{z_{n}} \hat{G}_{n}(x) d x-\frac{\partial d_{2}\left(r_{n}\right)}{\partial r_{n}} p \int_{z_{n}}^{\infty}\left(x-z_{n}\right) \hat{g}_{n}(x) d x+\frac{\partial d_{2}\left(r_{n}\right)}{\partial r_{n}} c \int_{0}^{z_{n}} \hat{G}_{n}(x) d x=0,(30)
\end{aligned}
$$

(b) $\tilde{y}_{n}^{*}\left(z_{n}\right)=d_{1}\left(\tilde{r}_{n}^{*}\left(z_{n}\right)\right)+d_{2}\left(\tilde{r}_{n}^{*}\left(z_{n}\right)\right) z_{n}$.

Comparing with Equation (13) in Lemma 1, notice that in Equation (30) an extra term has been added which arises due to possible leftover inventory from period $n$. In Lemmas 3 and 4 in $\S 4$, for an additive and a multiplicative model, we will see how this term makes the optimal policies different from those for the perishable inventory case.

Let us define

$$
\begin{gathered}
I_{n}\left(z_{n}, \xi_{n+1}\left(z_{n}\right)\right)=\int_{0}^{z_{n}} V_{n+1}\left(x, \xi_{n+1}\left(z_{n}\right)\right) \hat{g}_{n}(x) d x+V_{n+1}^{c}\left(z_{n}, 0\right)\left[1-\hat{G}_{n}\left(z_{n}\right)\right] . \\
J\left(\hat{\pi}_{n}, \xi_{n}, z_{n}, \xi_{n+1}\left(z_{n}\right)\right)=M\left(\hat{\pi}_{n}, \xi_{n}, z_{n}, r_{n}\left(z_{n}\right)\right)+I_{n}\left(z_{n}, \xi_{n+1}\left(z_{n}\right)\right) .
\end{gathered}
$$


Similar to Equation (17), the marginal expected value of leftover inventory and information at $z_{n}$ can be written as

$$
\begin{aligned}
i_{n}\left(z_{n}, \xi_{n+1}\left(z_{n}\right)\right) & \equiv \frac{1}{1-\hat{G}_{n}\left(z_{n}\right)} \frac{d I_{n}\left(z_{n}, \xi_{n+1}\left(z_{n}\right)\right)}{d z_{n}} \\
& \equiv \frac{1}{1-\hat{G}_{n}\left(z_{n}\right)}\left[\int_{0}^{z_{n}} \frac{\partial V_{n+1}\left(x, \xi_{n+1}\left(z_{n}\right)\right)}{\partial z_{n}} \hat{g}_{n}(x) d x+\frac{d \tilde{I}_{n}\left(z_{n}\right)}{d z_{n}}\right]
\end{aligned}
$$

where

$$
\frac{d \tilde{I}_{n}\left(z_{n}\right)}{d z_{n}}=\frac{d V_{n+1}^{c}\left(z_{n}, 0\right)}{d z_{n}}\left[1-\hat{G}_{n}\left(z_{n}\right)\right]-\left(V_{n+1}^{c}\left(z_{n}, 0\right)-V_{n+1}\left(z_{n}, 0\right)\right) \hat{g}_{n}\left(z_{n}\right),
$$

for $n=1,2, \ldots, N-1$. Note that the first and the second term in Equation (33) represent the marginal expected value of leftover inventory and the marginal expected value of information respectively.

The following result gives an expression for $i_{n}\left(z_{n}, \xi_{n+1}\left(z_{n}\right)\right)$ which, compared to Theorem 1 , has additional terms because of holding inventory.

Theorem 2. (a) The marginal expected value of leftover inventory and information $i_{n}\left(z_{n}, \xi_{n+1}\left(z_{n}\right)\right)$ can be written as

$$
\begin{aligned}
i_{n}\left(z_{n}, \xi_{n+1}\left(z_{n}\right)\right)= & \frac{1}{1-\hat{G}_{n}\left(z_{n}\right)}\left[\int _ { 0 } ^ { z _ { n } } \left(c d_{2}\left(r_{n}\left(z_{n}\right)\right) I_{\left\{\tilde{z}_{n+1}^{* e}>z_{n}-x\right\}}\right.\right. \\
& \left.+\frac{\partial J\left(\pi_{n+1}, \xi_{n+1}\left(z_{n}\right), \tilde{z}_{n+1}^{* e}, \xi_{n+2}\left(\tilde{z}_{n+1}^{* e}\right)\right)}{\partial z_{n}} I_{\left\{\tilde{z}_{n+1}^{* e}=z_{n}-x\right\}}\right) \hat{g}_{n}(x) d x \\
& \left.+\hat{g}_{n}\left(z_{n}\right)\left(V_{n+1}\left(z_{n}, 0\right)-\left.V_{n+1}\left(z_{n}, 0\right)\right|_{z_{n+1}=\tilde{z}_{n+1}^{* c}}\right)\right]
\end{aligned}
$$

for $n=1,2, \ldots, N-1 .^{2}$

(b) The optimal stocking factor $\tilde{z}_{n}^{*}$ satisfies

$$
d_{2}\left(r_{n}\left(z_{n}\right)\right)\left(c+h^{\prime}\right) \hat{G}_{n}\left(z_{n}\right)=\left[1-\hat{G}_{n}\left(z_{n}\right)\right]\left[d_{2}\left(r_{n}\left(z_{n}\right)\right)\left(r_{n}\left(z_{n}\right)+p-c\right)+i_{n}\left(z_{n}, \xi_{n+1}\left(z_{n}\right)\right)\right],
$$

for $n=1,2, \ldots, N-1$.

From Theorem 2, under a condition on the order placement, we obtain the following structural property of the optimal stocking factor.

\footnotetext{
${ }^{2}$ The correction in this result from the earlier draft is due to an anonymous referee.
} 
Corollary 3. Assume that with stocking factor $z_{n}$ in a given period $n, n=1,2, \ldots, N-1$, an order will be placed in period $(n+1)$ for any demand realization in period $n$ (Condition $\left.C_{2}\right)$. Then

(a) $I_{n}\left(z_{n}, \xi_{n+1}\left(z_{n}\right)\right)$ is non-decreasing in $z_{n}, \quad n=1,2, \ldots, N-1$.

(b) And, if each single-period problem has a unique optimal myopic stocking factor $\tilde{z}_{n}^{B N}$, then the optimal stocking factor $\tilde{z}_{n}^{*}$ satisfies

$$
\tilde{z}_{n}^{*} \geq \tilde{z}_{n}^{B N}, n=1,2, \ldots, N-1, \quad \text { and } \quad \tilde{z}_{N}^{*}=\tilde{z}_{N}^{B N}
$$

From Equation (35) notice that if the Condition $C_{2}$ is not satisfied, which might be the case in general, then there will be sample paths with leftover inventory from period $n$ for which no order will be placed in period $(n+1)$ so that $\tilde{z}_{n+1}^{* e}=z_{n}-x$, and therefore, $\frac{\partial J\left(\pi_{n+1}, \xi_{n+1}\left(z_{n}\right), \tilde{z}_{n+1}^{* e}, \xi_{n+2}\left(\tilde{z}_{n+1}^{* e}\right)\right)}{\partial z_{n}} \leq 0$ on these sample paths that have positive probability. In such a case, $i_{n}\left(z_{n}, \xi_{n+1}\left(z_{n}\right)\right)$ is a sum of three terms, two of them are positive and one negative. One of the positive terms is the marginal value of information which corresponds to demand realizations that result in stockouts and the other one corresponds to demand realizations that lead to order placement in the next period even though there is leftover inventory. The negative term corresponds to demand realizations that lead to sufficient leftover inventory requiring no order placement in the next period. The net effect of these three terms can be either positive or negative and therefore, the structural result of Corollary 3 may fail to hold. Similar results are also observed independently by Lu, Song and Zhu (2005) and Chen and Plambeck (2004).

It is important to observe that the strong qualification of Condition $C_{2}$ is purely the impact of the nonperishability of inventory and not because of the pricing decision or demand censoring. Typically, under mild regularity conditions like $C_{1}$, the adverse impact of price can be overcome. However, the inventory effect persists even under full information on demand, even if we impose the buy-back assumption of Veinott (1965) and consider the backorder case.

Next, we compare $\tilde{z}_{n}^{*}$ with the optimal stocking factor corresponding to the inventory model which has full information on demand in the current period. If the realized value of period $n$ demand $D_{n}$ is 
exactly observed even when there is no leftover inventory at the end of the period, then we have $X_{n}=x_{n}$ and $\hat{\pi}_{n+1}\left(\theta \mid x_{n}\right) \equiv \pi_{n+1}\left(\theta \mid x_{n}\right)$ (compare with Equation (3)). The optimal future profit at period $n$ is given by

$$
I_{n}^{F}\left(z_{n}, \xi_{n+1}\left(z_{n}\right)\right) \equiv \int_{0}^{z_{n}} V_{n+1}^{F}\left(x, \xi_{n+1}\left(z_{n}\right)\right) \hat{g}_{n}(x) d x+\int_{z_{n}}^{\infty} V_{n+1}^{F}(x, 0) \hat{g}_{n}(x) d x
$$

for $n=1,2, \ldots, N-1$. The superscript $\mathrm{F}$ represents the full information scenario. Let $\tilde{z}_{n}^{F}$ denote the optimal stocking factor in period $n$ for this model. The following theorem shows that the inventory problem with censored information yields a higher optimal stocking factor than the full information model. This is so because acquisition of information on demand comes at the expense of a higher stocking factor than is the case when information is automatically guaranteed. Notice that this is a stronger result than comparing $\tilde{z}_{n}^{*}$ with the optimal stocking factor of a inventory system which has exact demand information in all periods up to and including the current period. Although analogous result holds also for perishable inventory, we will state the result for the non-perishable inventory case.

Theorem 3. $\tilde{z}_{n}^{*} \geq \tilde{z}_{n}^{F}$, for $n=1,2, \ldots, N-1$.

We now investigate the optimal stocking factor of a period with respect to $N$ for the non-perishable inventory model. Let $\tilde{z}_{n, N}^{*}, V_{n, N}\left(\hat{\pi}_{n}, \xi_{n}\right)$, and $I_{n, N}\left(z_{n}, \xi_{n+1}\left(z_{n}\right)\right)$ respectively denote the optimal stocking factor, the optimal profit, and the optimal future profit (refer to Equations (29) and (31)) in period $n$ of an $N$-period problem. Also let $\tilde{I}_{n, N}\left(z_{n}\right)$ represent $\tilde{I}_{n}\left(z_{n}\right)$ (refer to Equation (34)) in this case. For a two-period problem, if an order is placed in the second period, then using Corollary 3(a) and arguments similar to those leading to Equation (23) we can show $\tilde{z}_{1,2}^{*} \geq \tilde{z}_{1,1}^{*}$; otherwise, this inequality may not hold. To compare $\tilde{z}_{n, N+1}^{*}$ and $\tilde{z}_{n, N}^{*}$, for any $n<N$, in inventory problems with horizon lengths of more than two periods, we write from Equation (33),

$$
\begin{aligned}
& \frac{d I_{n, N+1}\left(z_{n}, \xi_{n+1}\left(z_{n}\right)\right)}{d z_{n}}-\frac{d I_{n, N}\left(z_{n}, \xi_{n+1}\left(z_{n}\right)\right)}{d z_{n}} \\
= & \left(\int_{0}^{z_{n}} \frac{\partial V_{n+1, N+1}\left(x, \xi_{n+1}\left(z_{n}\right)\right)}{\partial z_{n}} \hat{g}_{n}(x) d x-\int_{0}^{z_{n}} \frac{\partial V_{n+1, N}\left(x, \xi_{n+1}\left(z_{n}\right)\right)}{\partial z_{n}} \hat{g}_{n}(x) d x\right)
\end{aligned}
$$




$$
+\left(\frac{d \tilde{I}_{n, N+1}\left(z_{n}\right)}{d z_{n}}-\frac{d \tilde{I}_{n, N}\left(z_{n}\right)}{d z_{n}}\right)
$$

From Theorem 2(a) we observe that the net effect of the two terms inside the first parethesis of Equation (38) can be positive or negative in general, and is zero only in the special case when Condition $C_{2}$ of Corollary 3 is satisfied. On the other hand, using Theorem 2(a) again and analogously to the perishable inventory case, we see that $\left(\frac{d \tilde{I}_{n, N+1}\left(z_{n}\right)}{d z_{n}}-\frac{d \tilde{I}_{n, N}\left(z_{n}\right)}{d z_{n}}\right)$ can be either positive or negative. Therefore, the inequality $\tilde{z}_{n, N+1}^{*} \geq \tilde{z}_{n, N}^{*}$ may not hold also for non-perishable products even under Condition $C_{2}$.

To complete the analysis, let us also examine the behavior of the optimal stocking factor of a period with respect to $N$ for the full information model. Let $\tilde{z}_{n, N}^{F}$ and $I_{n, N}^{F}\left(z_{n}, \xi_{n+1}\left(z_{n}\right)\right)$ respectively denote the optimal stocking factor and the optimal future profit (refer to Equation (37)) in period $n$ of an $N$-period problem. From the proofs of Theorem 2(a) and Theorem 3 we can reach the following conclusions. For a two-period problem, if an order is placed in the second period, then $\frac{d I_{1,2}^{F}\left(z_{1}, \xi_{2}\left(z_{1}\right)\right)}{d z_{1}} \geq 0$, which implies $\tilde{z}_{1,2}^{F} \geq \tilde{z}_{1,1}^{F}$; otherwise, this inequality need not hold. If the duration of the problems are longer than two periods, then assuming Condition $C_{2}$ for the full information model, we have $\frac{d I_{n, N+1}^{F}\left(z_{n}, \xi_{n+1}\left(z_{n}\right)\right)}{d z_{n}}-$ $\frac{d I_{n, N}^{F}\left(z_{n}, \xi_{n+1}\left(z_{n}\right)\right)}{d z_{n}}=0$, which implies $\tilde{z}_{n, N+1}^{F}=\tilde{z}_{n, N}^{F}$; otherwise, this equality may not hold in general. Furthermore, if the inventory is perishable in the full information model, then we have $\frac{d I_{n, N}^{F}\left(z_{n}, 0\right)}{d z_{n}}=0$, which means $z_{n, N+1}^{F}=z_{n, N}^{F}$ for all $N$, where $z_{n, N}^{F}$ denotes the optimal stocking factor in period $n$ for the perishable inventory case. Table 1 summarizes the properties of the optimal stocking factor of period $n$ with respect to the length of the problem horizon for different inventory models. It is worth noting that the above results hold also for the observed lost sales inventory models with either known or unknown demand distribution because these are only special cases of our full information model.

We now ask the following question along the same line as Petruzzi and Dada (1999) for our nonperishable inventory model. How would the optimal stocking factors behave if a salvage market existed such that the leftover inventory from a period can be sold at the beginning of the next period, before placing the order? If all the leftovers are salvaged, then arguing similarly to Petruzzi and Dada (1999), 


\begin{tabular}{|c|c|c|}
\hline Inventory & Full Information & Censored Information \\
\hline \hline \multirow{2}{*}{ Perishable } & $z_{n, N+1}^{F}=z_{n, N}^{F} \forall N$ and all $n<N$ & $z_{1,2}^{*} \geq z_{1,1}^{*} ;$ \\
\hline \hline \multirow{2}{*}{ Non-perishable } & $\tilde{z}_{1,2}^{F} \geq \tilde{z}_{1,1}^{F}, \tilde{z}_{n, N+1}^{F}=\tilde{z}_{n, N}^{F} \forall N \geq 2 \forall n<N$ & $\tilde{z}_{1,2}^{*} \geq \tilde{z}_{1,1}^{*} \quad$ under Condition $C_{2} ;$ \\
& Inder Condition $C_{2} ;$ & \\
& Indeterminate $\forall N \geq 1 \quad$ otherwise & Indeterminate $\forall N \geq 1$ otherwise \\
\hline
\end{tabular}

Table 1: Behavior of the optimal stocking factor of a period for different $N$ and various inventory models

we would conclude that, in the case of the full information model, the optimal stocking factors are obtained by solving single-period (myopic) problems with the updated distribution function. But this myopic property does not hold if the demand observations are censored. In this case, the optimal stocking factors behave as if the inventory is perishable with some adjustments of the cost/revenue parameters. Also the behavior of the optimal stocking factor of a period with respect to $N$ is similar to that of the perishable inventory case as described in Table 1. Although, as argued by Petruzzi and Dada (1999), the existence of a salvage market is unnecessary when the demand distribution is known, it can be very important when updating of the demand distribution is required. Consider the following scenario. Suppose the prior distribution at the beginning of a period indicated a high demand resulting in a large order quantity. But in reality actual demand turns out to be low. If this happens, the posterior distribution may readjust itself to suggest a low demand for the next period. In such a case, it is worthwhile to have a salvage market where some or all of the leftover inventories could be sold. 
The questions now arise how much of the leftover inventory should be salvaged? Should we salvage all or part of it? For a two-period problem as considered in Petruzzi and Dada (2001), it is possible to salvage any inventory that is excess over the second period's optimal stocking quantity because this can be calculated myopically. But deciding on how much of the leftover inventory should be salvaged in each period of a general $N$-period problem is a difficult issue because the optimal stocking decisions are no longer myopic.

\section{The Cases of Additive and Multiplicative Demands}

Now that we have provided a broad view of the censored newsvendor problem, to glean sharper insights we consider the specific cases of additive and multiplicative demands. We find that for a given stocking factor $z_{n}$, the optimal policies in the additive model have identical properties for both perishable and non-perishable inventory cases, but behave differently in the multiplicative model.

\subsection{The Additive Model}

The following lemma gives the optimal pricing and ordering policies for the additive demand model obtained from Equation (1) by letting $d_{1}\left(r_{n}\right)=a-b r_{n}(a>0, b>0)$ and $d_{2}\left(r_{n}\right)=1$.

Lemma 3. In the additive demand model, for both the cases of perishable and non-perishable inventory,

(a) $r_{n}^{*}\left(z_{n}\right)=\frac{a+b c+z_{n}-\int_{0}^{z_{n}} \hat{G}_{n}(x) d x}{2 b}$

(b) $y_{n}^{*}\left(z_{n}\right)=\frac{a-b c+z_{n}+\int_{0}^{z_{n}} \hat{G}_{n}(x) d x}{2}$

(c) $r_{n}^{*}\left(z_{n}\right)$ increasing and concave, whereas $y_{n}^{*}\left(z_{n}\right)$ increasing and convex in $z_{n}$,

(d) As $z_{n} \rightarrow \infty, r_{n}^{*}\left(z_{n}\right)$ uniformly converges to the optimal riskless price $\frac{a+b c+E\left[X_{n}\right]}{2 b}$.

Lemma 3 leads to the following consequences of Corollaries 1(b) and 3(b) for the additive demand model. To state the result we introduce some notation. For the case of perishable inventory, let us denote 
$r_{n}^{*}=r_{n}^{*}\left(z_{n}^{*}\right), r_{n}^{B N}=r_{n}^{*}\left(z_{n}^{B N}\right)$, and $y_{n}^{*}=y_{n}^{*}\left(z_{n}^{*}\right), y_{n}^{B N}=y_{n}^{*}\left(z_{n}^{B N}\right) ;$ and for the case of non-perishable inventory, let us denote $\tilde{r}_{n}^{*}=r_{n}^{*}\left(\tilde{z}_{n}^{*}\right), \tilde{r}_{n}^{B N}=r_{n}^{*}\left(\tilde{z}_{n}^{B N}\right)$, and $\tilde{y}_{n}^{*}=y_{n}^{*}\left(\tilde{z}_{n}^{*}\right), \tilde{y}_{n}^{B N}=y_{n}^{*}\left(\tilde{z}_{n}^{B N}\right)$.

Theorem 4. If each single-period problem has a unique optimal myopic stocking factor $z_{n}^{B N}$ (resp. $\tilde{z}_{n}^{B N}$ ) in the additive model for the case of perishable inventory (resp. for the case of non-perishable inventory with Condition $C_{2}$ ), then

$$
\begin{aligned}
& r_{n}^{*} \geq r_{n}^{B N}\left(\text { resp. } \quad \tilde{r}_{n}^{*} \geq \tilde{r}_{n}^{B N}\right), \quad \text { and } y_{n}^{*} \geq y_{n}^{B N}\left(\text { resp. } \quad \tilde{y}_{n}^{*} \geq \tilde{y}_{n}^{B N}\right), n=1,2, \ldots, N-1 ; \\
& r_{N}^{*}=r_{N}^{B N}\left(\text { resp. } \quad \tilde{r}_{N}^{*}=\tilde{r}_{N}^{B N}\right), \quad \text { and } \quad y_{N}^{*}=y_{N}^{B N}\left(\text { resp. } \quad \tilde{y}_{N}^{*}=\tilde{y}_{N}^{B N}\right) .
\end{aligned}
$$

Theorem 4 is an insightful result. It establishes that for the additive demand case, if lost sales are unobservable, the optimal policy for the newsvendor is not only to order higher quantities from the supplier but also to charge higher retail prices from the consumer than in the case where there is no demand learning. In other words, long-term information acquisition and optimality come at the expense of short-term costs to the retailer (higher leftovers) as well as to the consumer (higher retail prices).

Furthermore, Theorem 3 and Lemma 3 together imply the following result. To describe it, let us denote $r_{n}^{F}=r_{n}^{*}\left(z_{n}^{F}\right)$ and $y_{n}^{F}=y_{n}^{*}\left(z_{n}^{F}\right)$ for the case of perishable inventory; and $\tilde{r}_{n}^{F}=r_{n}^{*}\left(\tilde{z}_{n}^{F}\right)$ and $\tilde{y}_{n}^{F}=y_{n}^{*}\left(\tilde{z}_{n}^{F}\right)$ for the case of non-perishable inventory. The result can be interpreted in the same way as Theorem 4 .

Theorem 5. For the additive model,

$$
r_{n}^{*} \geq r_{n}^{F}\left(\text { resp. } \quad \tilde{r}_{n}^{*} \geq \tilde{r}_{n}^{F}\right), \quad \text { and } \quad y_{n}^{*} \geq y_{n}^{F}\left(\text { resp. } \quad \tilde{y}_{n}^{*} \geq \tilde{y}_{n}^{F}\right), \quad n=1,2, \ldots, N-1 .
$$

\subsection{The Multiplicative Model}

The following lemma describes the optimal policies for the multiplicative demand model given by Equation (1) with $d_{1}\left(r_{n}\right)=0$ and $d_{2}\left(r_{n}\right)=a r_{n}^{-b}(a>0, b \geq 2)$. 
Lemma 4. For the multiplicative demand model,

(a) $r_{n}^{*}\left(z_{n}\right)=\frac{b}{b-1}\left[c+\frac{(c-h) \int_{0}^{z_{n}} \hat{G}_{n}(x) d x+p \int_{z_{n}}^{\infty}\left(x-z_{n}\right) \hat{g}_{n}(x) d x}{\int_{0}^{z_{n}}\left(1-\hat{G}_{n}(x)\right) d x}\right] \quad$ (for the case of perishable inventory) and $r_{n}^{*}\left(z_{n}\right)=\frac{b}{b-1}\left[c+\frac{h^{\prime} \int_{0}^{z_{n}} \hat{G}_{n}(x) d x+p \int_{z_{n}}^{\infty}\left(x-z_{n}\right) \hat{g}_{n}(x) d x}{\int_{0}^{z_{n}}\left(1-\hat{G}_{n}(x)\right) d x}\right]$ (for the case of non-perishable inventory),

(b) $y_{n}^{*}\left(z_{n}\right)=a\left(r_{n}^{*}\left(z_{n}\right)\right)^{-b} z_{n}$,

(c) Let $z_{n}^{T}$ be defined such that $(c-h) \int_{0}^{z_{n}^{T}} x \hat{g}_{n}(x) d x-p\left(1-\hat{G}_{n}\left(z_{n}^{T}\right)\right) E\left[X_{n}\right]=0$ for the perishable inventory case (resp. $h^{\prime} \int_{0}^{z_{n}^{T}} x \hat{g}_{n}(x) d x-p\left(1-\hat{G}_{n}\left(z_{n}^{T}\right)\right) E\left[X_{n}\right]=0$ for the non-perishable inventory case). Then $r_{n}^{*}\left(z_{n}\right)$ is decreasing and convex for $z_{n}<z_{n}^{T}$ and increasing for $z_{n}>z_{n}^{T}$. Also, $y_{n}^{*}\left(z_{n}\right)$ is increasing for $z_{n}<z_{n}^{T}$, and for $z_{n}>z_{n}^{T}$, $y_{n}^{*}\left(z_{n}\right)$ is increasing if $0<\frac{d r_{n}^{*}\left(z_{n}\right)}{d z_{n}}<\frac{r_{n}^{*}\left(z_{n}\right)}{b z_{n}}$ and decreasing if $\frac{d r_{n}^{*}\left(z_{n}\right)}{d z_{n}}>\frac{r_{n}^{*}\left(z_{n}\right)}{b z_{n}}$.

From Lemma $4(\mathrm{a})$ we see that $r_{n}^{*}\left(z_{n}\right) \geq \frac{b c}{b-1}$, the optimal riskless price. Thus, for the multiplicative model, the riskless price is a lower bound for the optimal price. On the other hand, for the additive model, from Lemma 3 we observe that the riskless price is an upper bound as well as a limiting value for the optimal price. Figures 2 and 3 present typical descriptions of $r_{n}^{*}\left(z_{n}\right)$ and $y_{n}^{*}\left(z_{n}\right)$ graphically.

Table 2 summarizes the similarities and differences of the relationships between the optimal and myopic policies for the additive and the multiplicative models. We see that operational decisions can be easily interpreted from the learning effect of demand for the additive case, whereas it is not easy to interpret these for the multiplicative case. From Lemmas 3 and 4 we note that the optimal pricing and ordering decisions depend on the expected lost sales and the parameters $p, h$, and $h^{\prime}$ for the multiplicative demand case but not for the additive demand case.

\section{Conclusion}

This paper takes a general approach to studying dynamic pricing and ordering decisions for a newsvendor model with unobservable lost sales and stochastic demand involving an unknown parameter. We develop 


\begin{tabular}{|c|c|c|}
\hline Variable & Additive Model & Multiplicative Model \\
\hline \hline$z_{n}$ & $z_{n}^{*} \geq z_{n}^{B N}$ & $z_{n}^{*} \geq z_{n}^{B N}$ \\
\hline \hline$r_{n}$ & $r_{n}^{*} \geq r_{n}^{B N}$ & $r_{n}^{*} \geq r_{n}^{B N}$ always if $p=0 ;$ \\
\hline & & $r_{n}^{*} \geq r_{n}^{B N}$ if $z_{n}^{*}, z_{n}^{B N} \geq z_{n}^{T}$, \\
\hline & & $r_{n}^{*} \leq r_{n}^{B N}$ if $z_{n}^{*}, z_{n}^{B N} \leq z_{n}^{T}$, \\
\hline \hline$y_{n}$ & $y_{n}^{*} \geq y_{n}^{B N}$ & $y_{n}^{*} \geq y_{n}^{B N}$ if $z_{n}^{*}, z_{n}^{B N} \leq z_{n}^{T}$, \\
\hline & & Indeterminate if $z_{n}^{B N} \leq z_{n}^{T} \leq z_{n}^{*}$ \\
\hline
\end{tabular}

Table 2: Comparison between additive and multiplicative demand models (under Condition $C_{2}$ in the case of non-perishable inventory) 
a unified framework to analyze a very general class of demand models for both perishable and nonperishable products. The optimization problem of jointly pricing and ordering is first reduced to a single-variable problem in the stocking factor. Optimal prices and order quantities are then evaluated myopically as unique functions of the stocking factor. We develop important identities for the marginal expected value of information and leftover inventory. As consequences of these identities, we show that the optimal stocking factor at every intermediate decision epoch is higher than the Bayesian myopic stocking factor when inventory is perishable. If inventory is non-perishable, the same result holds only under a strong condition on the order placement. This identity also helps to understand why the optimal stocking factor of a period in the censored demand case may not increase with respect to the problem lengths. Finally, the implications of the results are discussed in the context of an additive and a multiplicative demand model. For the additive case, we show when demand is censored the optimal policy would be to order higher amount as well as charge higher retail prices than both the Bayesian myopic model and the full information model. We find that the optimal policies in the additive model are identical for both perishable and non-perishable inventories, but they differ in the multiplicative model. We also compare the optimal and myopic policies for these two models.

This paper establishes new results as well as generalizes some of the existing results in the literature. For example, the structural result stated in Corollary 1(b) has been proved in Ding, Puterman and Bisi (2002) for a model with a fixed retail price. But to obtain this result they assumed that the probability density function $f(x \mid \theta)$ of $X_{n}$ is likelihood-ratio increasing in $\theta$. Our paper shows that this assumption is not required. Moreover, when specialized to the case of the fixed retail price, results in this paper hold also for their model.

Petruzzi and Dada (2001) have obtained some results similar to this paper. However, they consider only a two-period problem with an additive demand function. Even for this simpler model, the results are obtained under the assumption that the updated distribution function of $X_{2}$ is non-increasing in the first-period stocking factor if the demand is censored in the first period, that is, $\frac{\partial G_{2}^{c}\left(x \mid z_{1}\right)}{\partial z_{1}} \leq 0$. While 
working on this paper we realized that this condition is not correct. An appropriate condition should have been $\frac{\partial \hat{G}_{2}^{U}\left(x \mid z_{1}\right)}{\partial z_{1}} \leq 0$, that is, the updated marginal distribution function of $X_{2}$ (after unconditioning w.r.t. $\left.X_{1}\right)$ is non-increasing in the first-period stocking factor irrespective of the demand observation in the first period. From Proposition 1 we observe that $\frac{\partial \hat{G}_{2}^{U}\left(x \mid z_{1}\right)}{\partial z_{1}}=0$ is always true and therefore, results in Petruzzi and Dada (2001) hold without any assumption on $\hat{G}_{2}^{U}\left(x \mid z_{1}\right)$.

Notice that even though we have assumed the probability distributions of $X_{n}, n=1,2, \ldots, N$, for given $\theta$ are iid over periods, the unconditional distributions of $X_{n} \mathrm{~s}$ are neither independent nor identical over periods because the updated distributions of $X_{n} \mathrm{~s}$ are based on all previous demand realizations which directly influence the prior updates of $\boldsymbol{\theta}$. Moreover, since we do not assume that the prior distribution of $\boldsymbol{\theta}$ should be conjugate to the demand distribution, the updated distribution of $X_{n}$ can be different over periods. This generalization is essential for censored demand models where most of the distributions do not have any conjugate prior, one exception being the family of Newsboy distributions with a gamma prior (Braden and Freimer 1991). Thus, our model has practical significance in many businesses, e.g., fashion goods, new videos and CDs, high-tech industries like DRAM and computer manufacturing where products are characterized by short shelf life and volatile markets.

The model and methodology considered in this paper can also be extended to study infinite horizon problems where there is a possibility that the true demand distribution will be completely revealed once the underlying demand process becomes stable with a steady state distribution. This approach is suitable for modeling the demand of a basic commodity type of product which has been launched in the market very recently and is going to have a long life cycle. The interesting issues that can be addressed in this context are whether the optimal policies converge. And, if they do, what are the limiting policies, what are the convergence rates and so on. For the observable demand case, these issues have been studied by Scarf (1959) for the exponential family. However, due to the complexity associated with the censored demand case where the updated distributions in any period depend on all past actions, these are challenging research questions which require seperate investigation on their own. 
Although not in the scope of this paper, finding efficient ways of computing the optimal policies and comparing their performance against the Bayesian myopic policies would be an interesting problem to pursue. We believe that Equation (18) in Theorem 1(a) would play a key role to this issue. We leave this for future research.

\section{Appendix}

Proof of Lemma 1. (a) Notice that the last two terms on the right hand side of the optimality equation (9) are independent of $r_{n}$. Moreover, for each given value of $z_{n}, M\left(\hat{\pi}_{n}, z_{n}, r_{n}\right)$ (refer to Equation (8)) is concave in $r_{n}$. Therefore, the optimal selling price is obtained by solving for $r_{n}$ from $\frac{\partial M\left(\hat{\pi}_{n}, z_{n}, r_{n}\right)}{\partial r_{n}}=$ 0, which leads to Equation (13).

(b) Follows immediately from Equation (2) after replacing $r_{n}$ by $r_{n}^{*}\left(z_{n}\right)$.

Proof of Theorem 1. (a) We will prove the result for $n$, where $n=1,2, \ldots, N-2$. For $n=N-1$, the result follows similarly with the boundary condition $V_{N+1}\left(\hat{\pi}_{N+1}\right)=0$ for all $\hat{\pi}_{N+1}$.

From Equation (17) we recall

$$
i_{n}\left(z_{n}\right)=\frac{d V_{n+1}^{c}\left(z_{n}\right)}{d z_{n}}-\left(V_{n+1}^{c}\left(z_{n}\right)-V_{n+1}\left(z_{n}\right)\right) \frac{\hat{g}_{n}\left(z_{n}\right)}{1-\hat{G}_{n}\left(z_{n}\right)}, \quad \text { for } n=1,2, \ldots, N-1
$$

Rearranging terms in Equation (39) we write

$$
i_{n}\left(z_{n}\right)=V_{n+1}\left(z_{n}\right) \frac{\hat{g}_{n}\left(z_{n}\right)}{1-\hat{G}_{n}\left(z_{n}\right)}-\left[V_{n+1}^{c}\left(z_{n}\right) \frac{\hat{g}_{n}\left(z_{n}\right)}{1-\hat{G}_{n}\left(z_{n}\right)}-\frac{d V_{n+1}^{c}\left(z_{n}\right)}{d z_{n}}\right]
$$

From (10) we note that

$$
z_{n+1}^{*}= \begin{cases}z_{n+1}^{* e} & \text { if } X_{n}=x_{n}<z_{n} \\ z_{n+1}^{* c} & \text { if } X_{n} \geq x_{n}=z_{n}\end{cases}
$$

Now from Equations (14)-(16), we write $V_{n+1}^{c}\left(z_{n}\right)$ and $V_{n+1}\left(z_{n}\right)$ as follows.

$$
V_{n+1}^{c}\left(z_{n}\right)=M\left(\pi_{n+1}^{c}, z_{n+1}^{* c}, r_{n+1}\left(z_{n+1}^{* c}\right)\right)+\int_{0}^{z_{n+1}^{* c}} V_{n+2}\left(x_{n+1}\right) g_{n+1}^{c}\left(x_{n+1} \mid z_{n}\right) d x_{n+1}
$$




$$
\begin{aligned}
& +V_{n+2}^{c}\left(z_{n+1}^{* c}\right)\left[1-G_{n+1}^{c}\left(z_{n+1}^{* c} \mid z_{n}\right)\right] \\
= & d_{1}\left(r_{n+1}\left(z_{n+1}^{* c}\right)\right)\left(r_{n+1}\left(z_{n+1}^{* c}\right)-c\right)+d_{2}\left(r_{n+1}\left(z_{n+1}^{* c}\right)\right)\left[\left(r_{n+1}\left(z_{n+1}^{* c}\right)+p-c\right)\right. \\
& \left.-\left(r_{n+1}\left(z_{n+1}^{* c}\right)+p-h\right) G_{n+1}^{c}\left(z_{n+1}^{* c} \mid z_{n}\right)\right] z_{n+1}^{* c} \\
& +d_{2}\left(r_{n+1}\left(z_{n+1}^{* c}\right)\right)\left[\left(r_{n+1}\left(z_{n+1}^{* c}\right)-h\right) \int_{0}^{z_{n+1}^{* c}} x_{n+1} g_{n+1}^{c}\left(x_{n+1} \mid z_{n}\right) d x_{n+1}\right. \\
& \left.-p \int_{z_{n+1}^{* c}}^{\infty} x_{n+1} g_{n+1}^{c}\left(x_{n+1} \mid z_{n}\right) d x_{n+1}\right]+\int_{0}^{\infty} V_{n+2}\left(x_{n+1} \wedge z_{n+1}^{* c}\right) g_{n+1}^{c}\left(x_{n+1} \mid z_{n}\right) d x_{n+1},
\end{aligned}
$$

where, for $x_{n+1} \geq z_{n+1}^{* c}, V_{n+2}\left(x_{n+1} \wedge z_{n+1}^{* c}\right)=V_{n+2}\left(z_{n+1}^{* c}\right)$ which represents $V_{n+2}^{c}\left(z_{n+1}^{* c}\right)$. Similarly,

$$
\begin{aligned}
V_{n+1}\left(z_{n}\right)= & M\left(\pi_{n+1}, z_{n+1}^{* e}, r_{n+1}\left(z_{n+1}^{* e}\right)\right)+\int_{0}^{z_{n+1}^{* e}} V_{n+2}\left(x_{n+1}\right) g_{n+1}\left(x_{n+1} \mid z_{n}\right) d x_{n+1} \\
& +V_{n+2}^{c}\left(z_{n+1}^{* e}\right)\left[1-G_{n+1}\left(z_{n+1}^{* e} \mid z_{n}\right)\right] \\
= & d_{1}\left(r_{n+1}\left(z_{n+1}^{* e}\right)\right)\left(r_{n+1}\left(z_{n+1}^{* e}\right)-c\right)+d_{2}\left(r_{n+1}\left(z_{n+1}^{* e}\right)\right)\left[\left(r_{n+1}\left(z_{n+1}^{* e}\right)+p-c\right)\right. \\
& \left.-\left(r_{n+1}\left(z_{n+1}^{* e}\right)+p-h\right) G_{n+1}\left(z_{n+1}^{* e} \mid z_{n}\right)\right] z_{n+1}^{* e} \\
& +d_{2}\left(r_{n+1}\left(z_{n+1}^{* e}\right)\right)\left[\left(r_{n+1}\left(z_{n+1}^{* e}\right)-h\right) \int_{0}^{z_{n+1}^{* e}} x_{n+1} g_{n+1}\left(x_{n+1} \mid z_{n}\right) d x_{n+1}\right. \\
& \left.-p \int_{z_{n+1}^{* e}}^{\infty} x_{n+1} g_{n+1}\left(x_{n+1} \mid z_{n}\right) d x_{n+1}\right]+\int_{0}^{\infty} V_{n+2}\left(x_{n+1} \wedge z_{n+1}^{* e}\right) g_{n+1}\left(x_{n+1} \mid z_{n}\right) d x_{n+1},
\end{aligned}
$$

where, for $x_{n+1} \geq z_{n+1}^{* e}, V_{n+2}\left(x_{n+1} \wedge z_{n+1}^{* e}\right)=V_{n+2}\left(z_{n+1}^{* e}\right)$ which represents $V_{n+2}^{c}\left(z_{n+1}^{* e}\right)$.

Let $\left.M\left(\pi_{n+1}, z_{n+1}, r_{n+1}\left(z_{n+1}\right)\right)\right|_{z_{n+1}=z_{n+1}^{* c}}$ denote the expected profit at period $(n+1)$ when $M\left(\pi_{n+1}, z_{n+1}, r_{n+1}\left(z_{n+1}\right)\right)$ is evaluated at $z_{n+1}=z_{n+1}^{* c}$. Then

$$
\begin{aligned}
& \left.M\left(\pi_{n+1}, z_{n+1}, r_{n+1}\left(z_{n+1}\right)\right)\right|_{z_{n+1}=z_{n+1}^{* c}} \\
\equiv & d_{1}\left(r_{n+1}\left(z_{n+1}^{* c}\right)\right)\left(r_{n+1}\left(z_{n+1}^{* c}\right)-c\right)+d_{2}\left(r_{n+1}\left(z_{n+1}^{* c}\right)\right)\left[\left(r_{n+1}\left(z_{n+1}^{* c}\right)+p-c\right)\right. \\
& \left.-\left(r_{n+1}\left(z_{n+1}^{* c}\right)+p-h\right) G_{n+1}\left(z_{n+1}^{* c} \mid z_{n}\right)\right] z_{n+1}^{* c} \\
& +d_{2}\left(r_{n+1}\left(z_{n+1}^{* c}\right)\right)\left[\left(r_{n+1}\left(z_{n+1}^{* c}\right)-h\right) \int_{0}^{z_{n+1}^{* c}} x_{n+1} g_{n+1}\left(x_{n+1} \mid z_{n}\right) d x_{n+1}\right. \\
& \left.-p \int_{z_{n+1}^{* c}}^{\infty} x_{n+1} g_{n+1}\left(x_{n+1} \mid z_{n}\right) d x_{n+1}\right] .
\end{aligned}
$$


In the above equations, the density functions $g_{n+1}\left(x_{n+1} \mid z_{n}\right)$ and $g_{n+1}^{c}\left(x_{n+1} \mid z_{n}\right)$ are given by

$$
\begin{aligned}
& g_{n+1}\left(x_{n+1} \mid z_{n}\right)=\int_{\Theta} f\left(x_{n+1} \mid \theta\right) \pi_{n+1}\left(\theta \mid z_{n}\right) d \theta=\frac{\int_{\Theta} f\left(x_{n+1} \mid \theta\right) f\left(z_{n} \mid \theta\right) \hat{\pi}_{n}(\theta) d \theta}{\hat{g}_{n}\left(z_{n}\right)}, \\
& g_{n+1}^{c}\left(x_{n+1} \mid z_{n}\right)=\int_{\Theta} f\left(x_{n+1} \mid \theta\right) \pi_{n+1}^{c}\left(\theta \mid z_{n}\right) d \theta=\frac{\int_{\Theta} f\left(x_{n+1} \mid \theta\right)\left(1-F\left(z_{n} \mid \theta\right)\right) \hat{\pi}_{n}(\theta) d \theta}{1-\hat{G}_{n}\left(z_{n}\right)} .
\end{aligned}
$$

We now calculate $\frac{d V_{n+1}^{c}\left(z_{n}\right)}{d z_{n}}$. Using the chain rule of the partial derivatives and Leibniz's rule we get from Equation (41),

$$
\begin{aligned}
& \frac{d V_{n+1}^{c}\left(z_{n}\right)}{d z_{n}} \\
= & \frac{\partial V_{n+1}^{c}\left(z_{n}\right)}{\partial r_{n+1}\left(z_{n+1}^{* c}\right)} \frac{d r_{n+1}\left(z_{n+1}^{* c}\right)}{d z_{n}}+\frac{\partial V_{n+1}^{c}\left(z_{n}\right)}{\partial z_{n+1}^{* c}} \frac{d z_{n+1}^{* c}}{d z_{n}}+\frac{\partial V_{n+1}^{c}\left(z_{n}\right)}{\partial z_{n}} \\
= & \frac{\partial V_{n+1}^{c}\left(z_{n}\right)}{\partial z_{n}} \quad \text { (by the first-order optimality conditions) } \\
= & -d_{2}\left(r_{n+1}\left(z_{n+1}^{* c}\right)\right)\left(r_{n+1}\left(z_{n+1}^{* c}\right)+p-h\right) z_{n+1}^{* c} \frac{\partial G_{n+1}^{c}\left(z_{n+1}^{* c} \mid z_{n}\right)}{\partial z_{n}} \\
& +d_{2}\left(r_{n+1}\left(z_{n+1}^{* c}\right)\right)\left[\left(r_{n+1}\left(z_{n+1}^{* c}\right)-h\right) \int_{0}^{z_{n+1}^{* c}} x_{n+1} \frac{\partial g_{n+1}^{c}\left(x_{n+1} \mid z_{n}\right)}{\partial z_{n}} d x_{n+1}\right. \\
& \left.-p \int_{z_{n+1}^{* c}}^{\infty} x_{n+1} \frac{\partial g_{n+1}^{c}\left(x_{n+1} \mid z_{n}\right)}{\partial z_{n}} d x_{n+1}\right]+\int_{0}^{\infty} V_{n+2}\left(x_{n+1} \wedge z_{n+1}^{* c}\right) \frac{\partial g_{n+1}^{c}\left(x_{n+1} \mid z_{n}\right)}{\partial z_{n}} d x_{n+1} \\
& +\int_{0}^{\infty} \frac{\partial V_{n+2}\left(x_{n+1} \wedge z_{n+1}^{* c}\right)}{\partial z_{n}} g_{n+1}^{c}\left(x_{n+1} \mid z_{n}\right) d x_{n+1} .
\end{aligned}
$$

To simplify $i_{n}\left(z_{n}\right)$, we write the following equations. From Equation (45) we have

$$
G_{n+1}^{c}\left(z_{n+1}^{* c} \mid z_{n}\right)=\int_{0}^{z_{n+1}^{* c}} g_{n+1}^{c}\left(x_{n+1} \mid z_{n}\right) d x_{n+1}=\frac{\int_{\Theta} F\left(z_{n+1}^{* c} \mid \theta\right)\left(1-F\left(z_{n} \mid \theta\right)\right) \hat{\pi}_{n}(\theta) d \theta}{1-\hat{G}_{n}\left(z_{n}\right)},
$$

which gives by using (44),

$$
\frac{\partial G_{n+1}^{c}\left(z_{n+1}^{* c} \mid z_{n}\right)}{\partial z_{n}}=\frac{\hat{g}_{n}\left(z_{n}\right)}{1-\hat{G}_{n}\left(z_{n}\right)}\left[G_{n+1}^{c}\left(z_{n+1}^{* c} \mid z_{n}\right)-G_{n+1}\left(z_{n+1}^{* c} \mid z_{n}\right)\right]
$$

Also from Equation (45) we write

$$
\frac{\partial g_{n+1}^{c}\left(x_{n+1} \mid z_{n}\right)}{\partial z_{n}}=\frac{\hat{g}_{n}\left(z_{n}\right) \int_{\Theta} f\left(x_{n+1} \mid \theta\right)\left(1-F\left(z_{n} \mid \theta\right)\right) \hat{\pi}_{n}(\theta) d \theta}{\left[1-\hat{G}_{n}\left(z_{n}\right)\right]^{2}}-\frac{\int_{\Theta} f\left(x_{n+1} \mid \theta\right) f\left(z_{n} \mid \theta\right) \hat{\pi}_{n}(\theta) d \theta}{1-\hat{G}_{n}\left(z_{n}\right)} .
$$

Using (44) and (45), from (49) we get

$$
\frac{\partial g_{n+1}^{c}\left(x_{n+1} \mid z_{n}\right)}{\partial z_{n}}=\frac{\hat{g}_{n}\left(z_{n}\right)}{1-\hat{G}_{n}\left(z_{n}\right)}\left[g_{n+1}^{c}\left(x_{n+1} \mid z_{n}\right)-g_{n+1}\left(x_{n+1} \mid z_{n}\right)\right]
$$


Using (41)-(43) and (47)-(50), from (40) we get

$$
\begin{aligned}
i_{n}\left(z_{n}\right)= & V_{n+1}\left(z_{n}\right) \frac{\hat{g}_{n}\left(z_{n}\right)}{1-\hat{G}_{n}\left(z_{n}\right)}-\left[\left(\left.M\left(\pi_{n+1}, z_{n+1}, r_{n+1}\left(z_{n+1}\right)\right)\right|_{z_{n+1}=z_{n+1}^{* c}}\right) \frac{\hat{g}_{n}\left(z_{n}\right)}{1-\hat{G}_{n}\left(z_{n}\right)}\right. \\
+ & \left\{\left(\int_{0}^{\infty} V_{n+2}\left(x_{n+1} \wedge z_{n+1}^{* c}\right) g_{n+1}\left(x_{n+1} \mid z_{n}\right) d x_{n+1}\right) \frac{\hat{g}_{n}\left(z_{n}\right)}{1-\hat{G}_{n}\left(z_{n}\right)}\right. \\
& \left.\left.-\int_{0}^{\infty} \frac{\partial V_{n+2}\left(x_{n+1} \wedge z_{n+1}^{* c}\right)}{\partial z_{n}} g_{n+1}^{c}\left(x_{n+1} \mid z_{n}\right) d x_{n+1}\right\}\right] .
\end{aligned}
$$

From Equation (41), notice that $V_{n+2}\left(x_{n+1} \wedge z_{n+1}^{* c}\right)$ in Equation (51) is evaluated given that the demand is censored in period $n$, i.e., $X_{n} \geq z_{n}$, which is represented by $z_{n}^{c}$ below. To simplify Equation (51), we write $V_{n+2}\left(x_{n+1} \wedge z_{n+1}^{* c}\right)$ and $\frac{\partial V_{n+2}\left(x_{n+1} \wedge z_{n+1}^{* c}\right)}{\partial z_{n}}$ as follows.

$$
\begin{aligned}
V_{n+2}\left(x_{n+1} \wedge z_{n+1}^{* c}\right)= & d_{1}\left(r_{n+2}\left(z_{n+2}^{*}\right)\right)\left(r_{n+2}\left(z_{n+2}^{*}\right)-c\right)+d_{2}\left(r_{n+2}\left(z_{n+2}^{*}\right)\right)\left[\left(r_{n+2}\left(z_{n+2}^{*}\right)+p-c\right)\right. \\
& \left.-\left(r_{n+2}\left(z_{n+2}^{*}\right)+p-h\right) \hat{G}_{n+2}\left(z_{n+2}^{*} \mid x_{n+1} \wedge z_{n+1}^{* c}, z_{n}^{c}\right)\right] z_{n+2}^{*} \\
& +d_{2}\left(r_{n+2}\left(z_{n+2}^{*}\right)\right)\left[\left(r_{n+2}\left(z_{n+2}^{*}\right)-h\right) \int_{0}^{z_{n+2}^{*}} x_{n+2} \hat{g}_{n+2}\left(x_{n+2} \mid x_{n+1} \wedge z_{n+1}^{* c}, z_{n}^{c}\right) d x_{n+2}\right. \\
& \left.-p \int_{z_{n+2}^{*}}^{\infty} x_{n+2} \hat{g}_{n+2}\left(x_{n+2} \mid x_{n+1} \wedge z_{n+1}^{* c}, z_{n}^{c}\right) d x_{n+2}\right] \\
& +\int_{0}^{\infty} V_{n+3}\left(x_{n+2} \wedge z_{n+2}^{*}\right) \hat{g}_{n+2}\left(x_{n+2} \mid x_{n+1} \wedge z_{n+1}^{* c}, z_{n}^{c}\right) d x_{n+2},
\end{aligned}
$$

where, for $x_{n+2} \geq z_{n+2}^{*}, V_{n+3}\left(x_{n+2} \wedge z_{n+2}^{*}\right)=V_{n+3}\left(z_{n+2}^{*}\right)$ which represents $V_{n+3}^{c}\left(z_{n+2}^{*}\right)$. To calculate $\frac{\partial V_{n+2}\left(x_{n+1} \wedge z_{n+1}^{* c}\right)}{\partial z_{n}}$, using the chain rule similarly to Equation (46) we get from Equation (52),

$$
\begin{aligned}
& \frac{\partial V_{n+2}\left(x_{n+1} \wedge z_{n+1}^{* c}\right)}{\partial z_{n}} \\
= & -d_{2}\left(r_{n+2}\left(z_{n+2}^{*}\right)\right)\left(r_{n+2}\left(z_{n+2}^{*}\right)+p-h\right) z_{n+2}^{*} \frac{\partial \hat{G}_{n+2}\left(z_{n+2}^{*} \mid x_{n+1} \wedge z_{n+1}^{* c}, z_{n}^{c}\right)}{\partial z_{n}} \\
& +d_{2}\left(r_{n+2}\left(z_{n+2}^{*}\right)\right)\left[\left(r_{n+2}\left(z_{n+2}^{*}\right)-h\right) \int_{0}^{z_{n+2}^{*}} x_{n+2} \frac{\partial \hat{g}_{n+2}\left(x_{n+2} \mid x_{n+1} \wedge z_{n+1}^{* c}, z_{n}^{c}\right)}{\partial z_{n}} d x_{n+2}\right. \\
& \left.-p \int_{z_{n+2}^{*}}^{\infty} x_{n+2} \frac{\partial \hat{g}_{n+2}\left(x_{n+2} \mid x_{n+1} \wedge z_{n+1}^{* c}, z_{n}^{c}\right)}{\partial z_{n}} d x_{n+2}\right] \\
& +\int_{0}^{\infty} V_{n+3}\left(x_{n+2} \wedge z_{n+2}^{*}\right) \frac{\partial \hat{g}_{n+2}\left(x_{n+2} \mid x_{n+1} \wedge z_{n+1}^{* c}, z_{n}^{c}\right)}{\partial z_{n}} d x_{n+2} \\
& +\int_{0}^{\infty} \frac{\partial V_{n+3}\left(x_{n+2} \wedge z_{n+2}^{*}\right)}{\partial z_{n}} \hat{g}_{n+2}\left(x_{n+2} \mid x_{n+1} \wedge z_{n+1}^{* c}, z_{n}^{c}\right) d x_{n+2} .
\end{aligned}
$$


In Equations (52) and (53), the density function $\hat{g}_{n+2}\left(x_{n+2} \mid x_{n+1} \wedge z_{n+1}^{* c}, z_{n}^{c}\right)$ is given by

$$
\begin{aligned}
\hat{g}_{n+2}\left(x_{n+2} \mid x_{n+1} \wedge z_{n+1}^{* c}, z_{n}^{c}\right) & =\int_{\Theta} f\left(x_{n+2} \mid \theta\right) \hat{\pi}_{n+2}\left(\theta \mid x_{n+1} \wedge z_{n+1}^{* c}, z_{n}^{c}\right) d \theta \\
& =\frac{\int_{\Theta} f\left(x_{n+2} \mid \theta\right) \hat{f}\left(x_{n+1} \wedge z_{n+1}^{* c} \mid \theta\right) \pi_{n+1}^{c}\left(\theta \mid z_{n}\right) d \theta}{\int_{\Theta} \hat{f}\left(x_{n+1} \wedge z_{n+1}^{* c} \mid \theta\right) \pi_{n+1}^{c}\left(\theta \mid z_{n}\right) d \theta} \\
& =\frac{\int_{\Theta} f\left(x_{n+2} \mid \theta\right) \hat{f}\left(x_{n+1} \wedge z_{n+1}^{* c} \mid \theta\right)\left(1-F\left(z_{n} \mid \theta\right)\right) \hat{\pi}_{n}(\theta) d \theta}{\int_{\Theta} \hat{f}\left(x_{n+1} \wedge z_{n+1}^{* c} \mid \theta\right)\left(1-F\left(z_{n} \mid \theta\right)\right) \hat{\pi}_{n}(\theta) d \theta},
\end{aligned}
$$

where

$$
\hat{f}\left(x_{n+1} \wedge z_{n+1}^{* c} \mid \theta\right)=\left\{\begin{array}{cc}
f\left(x_{n+1} \mid \theta\right) & \text { when } x_{n+1}<z_{n+1}^{* c} \\
1-F\left(z_{n+1}^{* c} \mid \theta\right) & \text { when } x_{n+1} \geq z_{n+1}^{* c} .
\end{array}\right.
$$

From Equation (54) we have

$$
\begin{aligned}
& \frac{\partial \hat{g}_{n+2}\left(x_{n+2} \mid x_{n+1} \wedge z_{n+1}^{* c}, z_{n}^{c}\right)}{\partial z_{n}} \\
= & \frac{\int_{\Theta} f\left(x_{n+2} \mid \theta\right) \hat{f}\left(x_{n+1} \wedge z_{n+1}^{* c} \mid \theta\right)\left(1-F\left(z_{n} \mid \theta\right)\right) \hat{\pi}_{n}(\theta) d \theta}{\left[\int_{\Theta} \hat{f}\left(x_{n+1} \wedge z_{n+1}^{* c} \mid \theta\right)\left(1-F\left(z_{n} \mid \theta\right)\right) \hat{\pi}_{n}(\theta) d \theta\right]^{2}}\left(\int_{\Theta} \hat{f}\left(x_{n+1} \wedge z_{n+1}^{* c} \mid \theta\right) f\left(z_{n} \mid \theta\right) \hat{\pi}_{n}(\theta) d \theta\right) \\
& -\frac{\int_{\Theta} f\left(x_{n+2} \mid \theta\right) \hat{f}\left(x_{n+1} \wedge z_{n+1}^{* c} \mid \theta\right) f\left(z_{n} \mid \theta\right) \hat{\pi}_{n}(\theta) d \theta}{\int_{\Theta} \hat{f}\left(x_{n+1} \wedge z_{n+1}^{* c} \mid \theta\right)\left(1-F\left(z_{n} \mid \theta\right)\right) \hat{\pi}_{n}(\theta) d \theta} \\
\equiv & \begin{cases}\frac{\partial g_{n+2}\left(x_{n+2} \mid x_{n+1}, z_{n}^{c}\right)}{\partial z_{n}} & \text { when } x_{n+1}<z_{n+1}^{* c} \\
\frac{\partial g_{n+2}^{c}\left(x_{n+2} \mid z_{n+1}^{* c}, z_{n}^{c}\right)}{\partial z_{n}} & \text { when } x_{n+1} \geq z_{n+1}^{* c} .\end{cases}
\end{aligned}
$$

where, from (55) we have

$$
\begin{gathered}
\frac{\partial g_{n+2}\left(x_{n+2} \mid x_{n+1}, z_{n}^{c}\right)}{\partial z_{n}}=\frac{1}{g_{n+1}^{c}\left(x_{n+1} \mid z_{n}\right)}\left[g_{n+2}\left(x_{n+2} \mid x_{n+1}, z_{n}^{c}\right)-g_{n+2}\left(x_{n+2} \mid x_{n+1}, z_{n}\right)\right] \times \\
g_{n+1}\left(x_{n+1} \mid z_{n}\right) \frac{\hat{g}_{n}\left(z_{n}\right)}{1-\hat{G}_{n}\left(z_{n}\right)} \\
\frac{\partial g_{n+2}^{c}\left(x_{n+2} \mid z_{n+1}^{* c}, z_{n}^{c}\right)}{\partial z_{n}}=\frac{1}{1-G_{n+1}^{c}\left(z_{n+1}^{* c} \mid z_{n}\right)}\left[g_{n+2}^{c}\left(x_{n+2} \mid z_{n+1}^{* c}, z_{n}^{c}\right)-g_{n+2}^{c}\left(x_{n+2} \mid z_{n+1}^{* c}, z_{n}\right)\right] \times \\
\left(1-G_{n+1}\left(z_{n+1}^{* c} \mid z_{n}\right)\right) \frac{\hat{g}_{n}\left(z_{n}\right)}{1-\hat{G}_{n}\left(z_{n}\right)}
\end{gathered}
$$

Now, using Equations (52), (53), (57), and (58), we simplify the last two terms on the right hand side of (51). To do this, we first analyze the terms that involve profit from period $(n+3)$ onwards. Let us write

$$
\int_{0}^{\infty}\left(\int_{0}^{\infty} V_{n+3}\left(x_{n+2} \wedge z_{n+2}^{*}\right) \frac{\partial \hat{g}_{n+2}\left(x_{n+2} \mid x_{n+1} \wedge z_{n+1}^{* c}, z_{n}^{c}\right)}{\partial z_{n}} d x_{n+2}\right) g_{n+1}^{c}\left(x_{n+1} \mid z_{n}\right) d x_{n+1}
$$




$$
\begin{aligned}
&= \int_{0}^{z_{n+1}^{* c}}\left(\int_{0}^{\infty} V_{n+3}\left(x_{n+2} \wedge z_{n+2}^{*}\right) \frac{\partial g_{n+2}\left(x_{n+2} \mid x_{n+1}, z_{n}^{c}\right)}{\partial z_{n}} d x_{n+2}\right) g_{n+1}^{c}\left(x_{n+1} \mid z_{n}\right) d x_{n+1} \\
&+\left(\int_{0}^{\infty} V_{n+3}\left(x_{n+2} \wedge z_{n+2}^{*}\right) \frac{\partial g_{n+2}^{c}\left(x_{n+2} \mid z_{n+1}^{* c}, z_{n}^{c}\right)}{\partial z_{n}} d x_{n+2}\right)\left(1-G_{n+1}^{c}\left(z_{n+1}^{* c} \mid z_{n}\right)\right) \\
&= {\left[\int_{0}^{z_{n+1}^{* c}}\left(\int_{0}^{\infty} V_{n+3}\left(x_{n+2} \wedge z_{n+2}^{*}\right)\left[g_{n+2}\left(x_{n+2} \mid x_{n+1}, z_{n}^{c}\right)-g_{n+2}\left(x_{n+2} \mid x_{n+1}, z_{n}\right)\right] d x_{n+2}\right) \times\right.} \\
&+\left(\int_{n+1}^{\infty}\left(x_{n+1} \mid z_{n}\right) d x_{n+1}\right] \frac{\hat{g}_{n}\left(z_{n}\right)}{1-\hat{G}_{n}\left(z_{n}\right)} \\
&= {\left.\left[\int_{0}^{\infty}\left(\int_{0}^{\infty} V_{n+3}\left(x_{n+2} \wedge z_{n+2}^{*}\right)\left[g_{n+2}^{c}\left(x_{n+2} \mid z_{n+1}^{* c}, z_{n}^{c}\right)-g_{n+2}^{c}\left(x_{n+2} \mid z_{n+1}^{* c}, z_{n}\right)\right] d x_{n+2}\right) \times \hat{g}_{n+2}\left(x_{n+2} \mid x_{n+1} \wedge z_{n+1}^{* c}, z_{n}^{c}\right)-\hat{g}_{n+2}\left(x_{n+2} \mid x_{n+1} \wedge z_{n+1}^{* c}, z_{n}\right)\right] d x_{n+2}\right) \times } \\
&\left.g_{n+1}\left(x_{n+1} \mid z_{n}\right) d x_{n+1}\right] \frac{\hat{g}_{n}\left(z_{n}\right)}{1-\hat{g}_{n}\left(z_{n}\right)} .
\end{aligned}
$$

Thus, the terms in (51) that represent expected profit from period $(n+3)$ onwards, together reduce to

$$
\begin{aligned}
& {\left[\int_{0}^{\infty}\left(\int_{0}^{\infty} V_{n+3}\left(x_{n+2} \wedge z_{n+2}^{*}\right) \hat{g}_{n+2}\left(x_{n+2} \mid x_{n+1} \wedge z_{n+1}^{* c}, z_{n}\right) d x_{n+2}\right) g_{n+1}\left(x_{n+1} \mid z_{n}\right) d x_{n+1}\right] \frac{\hat{g}_{n}\left(z_{n}\right)}{1-\hat{G}_{n}\left(z_{n}\right)}} \\
& -\int_{0}^{\infty}\left(\int_{0}^{\infty} \frac{\partial V_{n+3}\left(x_{n+2} \wedge z_{n+2}^{*}\right)}{\partial z_{n}} \hat{g}_{n+2}\left(x_{n+2} \mid x_{n+1} \wedge z_{n+1}^{* c}, z_{n}^{c}\right) d x_{n+2}\right) g_{n+1}^{c}\left(x_{n+1} \mid z_{n}\right) d x_{n+1} .
\end{aligned}
$$

Also, arguing similarly to (59) we can see that the expected profit at period $(n+2)$ in Equation (51) become

$$
\begin{aligned}
& \int_{0}^{\infty}\left(d_{1}\left(r_{n+2}\left(z_{n+2}^{*}\right)\right)\left(r_{n+2}\left(z_{n+2}^{*}\right)-c\right)+d_{2}\left(r_{n+2}\left(z_{n+2}^{*}\right)\right)\left[\left(r_{n+2}\left(z_{n+2}^{*}\right)+p-c\right)\right.\right. \\
& \left.-\left(r_{n+2}\left(z_{n+2}^{*}\right)+p-h\right) \hat{G}_{n+2}\left(z_{n+2}^{*} \mid x_{n+1} \wedge z_{n+1}^{* c}, z_{n}\right)\right] z_{n+2}^{*} \\
& +d_{2}\left(r_{n+2}\left(z_{n+2}^{*}\right)\right)\left[\left(r_{n+2}\left(z_{n+2}^{*}\right)-h\right) \int_{0}^{z_{n+2}^{*}} x_{n+2} \hat{g}_{n+2}\left(x_{n+2} \mid x_{n+1} \wedge z_{n+1}^{* c}, z_{n}\right) d x_{n+2}\right. \\
& \left.\left.-p \int_{z_{n+2}^{*}}^{\infty} x_{n+2} \hat{g}_{n+2}\left(x_{n+2} \mid x_{n+1} \wedge z_{n+1}^{* c}, z_{n}\right) d x_{n+2}\right]\right) g_{n+1}\left(x_{n+1} \mid z_{n}\right) d x_{n+1} \times \frac{\hat{g}_{n}\left(z_{n}\right)}{1-\hat{G}_{n}\left(z_{n}\right)} \\
\equiv & E_{\pi_{n+1}}\left(\left.M\left(\hat{\pi}_{n+2}, z_{n+2}^{*}, r_{n+2}\left(z_{n+2}^{*}\right)\right)\right|_{z_{n+1}=z_{n+1}^{* c}}\right) \frac{\hat{g}_{n}\left(z_{n}\right)}{1-\hat{G}_{n}\left(z_{n}\right)},
\end{aligned}
$$

where $\left.M\left(\hat{\pi}_{n+2}, z_{n+2}^{*}, r_{n+2}\left(z_{n+2}^{*}\right)\right)\right|_{z_{n+1}=z_{n+1}^{* c}}$ denote the expected profit at period $(n+2)$ given that the policy used in period $(n+1)$ is $z_{n+1}^{* c}$, i.e., the demand is censored in period $n$. $E_{\pi_{n+1}}$ represents expectation 
with respect to the density $g_{n+1}\left(x_{n+1} \mid z_{n}\right)$ (which is obtained from $\left.\pi_{n+1}\right)$ and also implies that $\hat{\pi}_{n+2}$ is based on $\pi_{n+1}$ (notice that not $\pi_{n+1}^{c}$ ) as is evident from the presence of $\hat{g}_{n+2}\left(x_{n+2} \mid x_{n+1} \wedge z_{n+1}^{* c}, z_{n}\right)$ in $(61)$.

Now, using (60) and (61), from (51) we get

$$
\begin{aligned}
i_{n}\left(z_{n}\right)= & V_{n+1}\left(z_{n}\right) \frac{\hat{g}_{n}\left(z_{n}\right)}{1-\hat{G}_{n}\left(z_{n}\right)}-\left[\left(\left.M\left(\pi_{n+1}, z_{n+1}, r_{n+1}\left(z_{n+1}\right)\right)\right|_{z_{n+1}=z_{n+1}^{* c}}\right) \frac{\hat{g}_{n}\left(z_{n}\right)}{1-\hat{G}_{n}\left(z_{n}\right)}\right. \\
& +E_{\pi_{n+1}}\left(\left.M\left(\hat{\pi}_{n+2}, z_{n+2}^{*}, r_{n+2}\left(z_{n+2}^{*}\right)\right)\right|_{z_{n+1}=z_{n+1}^{* c}}\right) \frac{\hat{g}_{n}\left(z_{n}\right)}{1-\hat{G}_{n}\left(z_{n}\right)} \\
& +\left\{\left(\int_{0}^{\infty}\left(\int_{0}^{\infty} V_{n+3}\left(x_{n+2} \wedge z_{n+2}^{*}\right) \hat{g}_{n+2}\left(x_{n+2} \mid x_{n+1} \wedge z_{n+1}^{* c}, z_{n}\right) d x_{n+2}\right) \times\right.\right. \\
& \left.g_{n+1}\left(x_{n+1} \mid z_{n}\right) d x_{n+1}\right) \frac{\hat{g}_{n}\left(z_{n}\right)}{1-\hat{G}_{n}\left(z_{n}\right)} \\
& \left.\left.-\int_{0}^{\infty}\left(\int_{0}^{\infty} \frac{\partial V_{n+3}\left(x_{n+2} \wedge z_{n+2}^{*}\right)}{\partial z_{n}} \hat{g}_{n+2}\left(x_{n+2} \mid x_{n+1} \wedge z_{n+1}^{* c}, z_{n}^{c}\right) d x_{n+2}\right) g_{n+1}^{c}\left(x_{n+1} \mid z_{n}\right) d x_{n+1}\right\}\right]
\end{aligned}
$$

Continuing the above analysis recursively while remembering that successive calculations are based on the sample paths which have been reached as a consequence of taking the policy $z_{n+1}^{* c}$ in period $(n+1)$, we finally get

$$
i_{n}\left(z_{n}\right)=\frac{\hat{g}_{n}\left(z_{n}\right)}{1-\hat{G}_{n}\left(z_{n}\right)}\left(V_{n+1}\left(z_{n}\right)-\left.V_{n+1}\left(z_{n}\right)\right|_{z_{n+1}=z_{n+1}^{* c}}\right)
$$

where

$$
\begin{aligned}
& \left.V_{n+1}\left(z_{n}\right)\right|_{z_{n+1}=z_{n+1}^{* c}} \\
= & \left.M\left(\pi_{n+1}, z_{n+1}, r_{n+1}\left(z_{n+1}\right)\right)\right|_{z_{n+1}=z_{n+1}^{* c}}+E_{\pi_{n+1}}\left(\left.M\left(\hat{\pi}_{n+2}, z_{n+2}^{*}, r_{n+2}\left(z_{n+2}^{*}\right)\right)\right|_{z_{n+1}=z_{n+1}^{* c}}\right) \\
& +\ldots+E_{\pi_{n+1}, \hat{\pi}_{n+2}, \ldots, \hat{\pi}_{N-1}}\left(\left.M\left(\hat{\pi}_{N}, z_{N}^{*}, r_{N}\left(z_{N}^{*}\right)\right)\right|_{z_{n+1}=z_{n+1}^{* c}}\right),
\end{aligned}
$$

and

$$
V_{n+1}\left(z_{n}\right)=\left.V_{n+1}\left(z_{n}\right)\right|_{z_{n+1}=z_{n+1}^{* e}} .
$$

This completes the proof.

(b) From Equation (16), we know that the optimal stocking factor satisfies

$$
z_{n}^{*} \in \arg \max _{z_{n}} J\left(\hat{\pi}_{n}, z_{n}\right), \text { for } n=1,2, \ldots, N-1
$$


Therefore, setting $\frac{d J\left(\hat{\pi}_{n}, z_{n}\right)}{d z_{n}}=0$, using (17), and rearranging terms we get Equation (19).

Proof of Corollary 1. (a) From Equations (17) and (63), we get

$$
\frac{d I_{n}\left(z_{n}\right)}{d z_{n}}=\hat{g}_{n}\left(z_{n}\right)\left(V_{n+1}\left(z_{n}\right)-\left.V_{n+1}\left(z_{n}\right)\right|_{z_{n+1}=z_{n+1}^{* c}}\right) .
$$

Since $V_{n+1}\left(z_{n}\right) \geq\left. V_{n+1}\left(z_{n}\right)\right|_{z_{n+1}=z_{n+1}^{* c}}$, the result follows.

(b) To prove $z_{n}^{*} \geq z_{n}^{B N}$ for $n=1,2, \ldots, N-1$, from Equation (15) we write

$$
\frac{d J\left(\hat{\pi}_{n}, z_{n}\right)}{d z_{n}}=\frac{d M\left(\hat{\pi}_{n}, z_{n}, r_{n}\left(z_{n}\right)\right)}{d z_{n}}+\frac{d I_{n}\left(z_{n}\right)}{d z_{n}} .
$$

By the assumption in the corollary we have $\left.\frac{d M\left(\hat{\pi}_{n}, z_{n}, r_{n}\left(z_{n}\right)\right)}{d z_{n}}\right|_{z_{n}=z_{n}^{B N}}=0$. Also, from part (a) we know $\frac{d I_{n}\left(z_{n}\right)}{d z_{n}} \geq 0$, at any $z_{n}$. Therefore, $\left.\frac{d J\left(\hat{\pi}_{n}, z_{n}\right)}{d z_{n}}\right|_{z_{n}=z_{n}^{B N}} \geq 0$, which implies $z_{n}^{*} \geq z_{n}^{B N}$, for $n=1,2, \ldots, N-1$.

For period $N$, since $V_{N+1}\left(\hat{\pi}_{N+1}\right)=0$, we have $V_{N}\left(\hat{\pi}_{N}\right)=\max _{z_{N} \in R_{+}}\left\{M\left(\hat{\pi}_{N}, z_{N}, r_{N}\left(z_{N}\right)\right)\right\}$. Hence, $z_{N}^{*}=z_{N}^{B N}$

Proof of Proposition 1. From Equation (21) we write

$$
\frac{\partial \hat{G}_{n}^{U}\left(x \mid z_{n-1}\right)}{\partial z_{n-1}}=\frac{\partial G_{n}^{c}\left(x \mid z_{n-1}\right)}{\partial z_{n-1}}\left[1-\hat{G}_{n-1}\left(z_{n-1}\right)\right]-\left(G_{n}^{c}\left(x \mid z_{n-1}\right)-G_{n}\left(x \mid z_{n-1}\right)\right) \hat{g}_{n-1}\left(z_{n-1}\right),
$$

for $n=2,3, \ldots, N$.

Similar to Equation (48), we can show

$$
\frac{\partial G_{n}^{c}\left(x \mid z_{n-1}\right)}{\partial z_{n-1}}\left[1-\hat{G}_{n-1}\left(z_{n-1}\right)\right]=\hat{g}_{n-1}\left(z_{n-1}\right)\left[G_{n}^{c}\left(x \mid z_{n-1}\right)-G_{n}\left(x \mid z_{n-1}\right)\right] .
$$

From (66) and (67) we get the desired result.

Proof of Lemma 2. (a) Notice that the second term on the right hand side of the optimality equation (29) depends on $r_{n}$ through the carried-over inventory $\xi_{n+1}\left(z_{n}\right)$. The optimal price is therefore obtained by solving $\frac{\partial J\left(\hat{\pi}_{n}, \xi_{n}, z_{n}, r_{n}\right)}{\partial r_{n}}=0$, which gives (30). Part (b) is obvious from Equation (2).

Proof of Theorem 2. (a) For the first term on the right hand side of Equation (33), we write

$$
V_{n+1}\left(x, \xi_{n+1}\left(z_{n}\right)\right)
$$




$$
\begin{aligned}
= & d_{1}\left(r_{n+1}\left(\tilde{z}_{n+1}^{* e}\right)\right)\left(r_{n+1}\left(\tilde{z}_{n+1}^{* e}\right)-c\right)+c \xi_{n+1}\left(z_{n}\right)+d_{2}\left(r_{n+1}\left(\tilde{z}_{n+1}^{* e}\right)\right)\left[\left(r_{n+1}\left(\tilde{z}_{n+1}^{* e}\right)+p-c\right)\right. \\
& \left.-\left(r_{n+1}\left(\tilde{z}_{n+1}^{* e}\right)+p+h^{\prime}\right) G_{n+1}\left(\tilde{z}_{n+1}^{* e} \mid x\right)\right] \tilde{z}_{n+1}^{* e} \\
& +d_{2}\left(r_{n+1}\left(\tilde{z}_{n+1}^{* e}\right)\right)\left[\left(r_{n+1}\left(\tilde{z}_{n+1}^{* e}\right)+h^{\prime}\right) \int_{0}^{\tilde{z}_{n+1}^{* e}} s g_{n+1}(s \mid x) d s-p \int_{\tilde{z}_{n+1}^{* e}}^{\infty} s g_{n+1}(s \mid x) d s\right] \\
& +\int_{0}^{\tilde{z}_{n+1}^{* e}} V_{n+2}\left(s, \xi_{n+2}\right) g_{n+1}(s \mid x) d s+V_{n+2}^{c}\left(\tilde{z}_{n+1}^{* e}, 0\right)\left[1-G_{n+1}\left(\tilde{z}_{n+1}^{* e} \mid x\right)\right] .
\end{aligned}
$$

Now, since $\xi_{n+1}\left(z_{n}\right)=d_{2}\left(r_{n}\left(z_{n}\right)\right)\left(z_{n}-x\right)$ for $X_{n}=x<z_{n}$, from Equation (68) we get

$$
\frac{\partial V_{n+1}\left(x, \xi_{n+1}\left(z_{n}\right)\right)}{\partial z_{n}}=\left\{\begin{array}{cl}
c d_{2}\left(r_{n}\left(z_{n}\right)\right) & \text { when } \tilde{z}_{n+1}^{* e}>z_{n}-x \\
\frac{\partial J\left(\pi_{n+1}, \xi_{n+1}\left(z_{n}\right), \tilde{z}_{n+1}^{* e}, \xi_{n+2}\left(\tilde{z}_{n+1}^{* e}\right)\right)}{\partial z_{n}} & \text { when } \tilde{z}_{n+1}^{* e}=z_{n}-x .
\end{array}\right.
$$

Moreover, we can mimic the proof of Theorem 1(a) to show that

$$
\frac{d \tilde{I}_{n}\left(z_{n}\right)}{d z_{n}}=\hat{g}_{n}\left(z_{n}\right)\left(V_{n+1}\left(z_{n}, 0\right)-\left.V_{n+1}\left(z_{n}, 0\right)\right|_{z_{n+1}=\tilde{z}_{n+1}^{* c}}\right) .
$$

Using Equations (69) and (70) in Equation (33), the result follows.

(b) The proof is similar to Theorem $1(\mathrm{~b})$.

Proof of Corollary 3. (a) The result follows from Equation (33) and Theorem 2(a).

(b) Using part (a) we can complete the proof along the same line of Corollary 1(b).

Proof of Theorem 3. From Equations (33) and (34), we know

$$
\frac{d I_{n}\left(z_{n}, \xi_{n+1}\left(z_{n}\right)\right)}{d z_{n}}=\int_{0}^{z_{n}} \frac{\partial V_{n+1}\left(x, \xi_{n+1}\left(z_{n}\right)\right)}{\partial z_{n}} \hat{g}_{n}(x) d x+\frac{d \tilde{I}_{n}\left(z_{n}\right)}{d z_{n}} .
$$

Applying Leibniz's rule, from Equation (37) we get

$$
\frac{d I_{n}^{F}\left(z_{n}, \xi_{n+1}\left(z_{n}\right)\right)}{d z_{n}}=\int_{0}^{z_{n}} \frac{\partial V_{n+1}^{F}\left(x, \xi_{n+1}\left(z_{n}\right)\right)}{\partial z_{n}} \hat{g}_{n}(x) d x
$$

We now compare Equations (71) and (72). Notice that $V_{n+1}\left(x, \xi_{n+1}\left(z_{n}\right)\right) \equiv V_{n+1}^{F}\left(x, \xi_{n+1}\left(z_{n}\right)\right)$ for $x<z_{n}$. Since $\frac{d \tilde{I}_{n}\left(z_{n}\right)}{d z_{n}} \geq 0$, we therefore have

$$
\frac{d I_{n}\left(z_{n}, \xi_{n+1}\left(z_{n}\right)\right)}{d z_{n}} \geq \frac{d I_{n}^{F}\left(z_{n}, \xi_{n+1}\left(z_{n}\right)\right)}{d z_{n}}
$$


Hence, $\tilde{z}_{n}^{*} \geq \tilde{z}_{n}^{F}$.

Proof of Lemma 3. $\quad$ Parts (a) and (b) follow from Lemmas 1 and 2.

(c) From part (a) notice that $\frac{d r_{n}^{*}\left(z_{n}\right)}{d z_{n}}=\frac{1-\hat{G}_{n}\left(z_{n}\right)}{2 b}>0$, and $\frac{d^{2} r_{n}^{*}\left(z_{n}\right)}{d z_{n}^{2}}=-\frac{\hat{g}_{n}\left(z_{n}\right)}{2 b}<0$. From part (b) notice that $\frac{d y_{n}^{*}\left(z_{n}\right)}{d z_{n}}=\frac{1+\hat{G}_{n}\left(z_{n}\right)}{2}>0$, and $\frac{d^{2} y_{n}^{*}\left(z_{n}\right)}{d z_{n}^{2}}=\frac{\hat{g}_{n}\left(z_{n}\right)}{2}>0$.

(d) Using the relation $E\left[X_{n}\right]=\int_{0}^{\infty}\left(1-\hat{G}_{n}(x)\right) d x$, the result follows from part (a).

Proof of Lemma 4. Parts (a) and (b) follow from Lemmas 1 and 2.

(c) We prove the result for the perishable inventory case. For non-perishable inventory, the proof is similar. From part (a) we can write

$$
\frac{d r_{n}^{*}\left(z_{n}\right)}{d z_{n}}=\frac{b}{b-1}\left[\frac{(c-h) \int_{0}^{z_{n}} x \hat{g}_{n}(x) d x-p\left(1-\hat{G}_{n}\left(z_{n}\right)\right) E\left[X_{n}\right]}{\left(\int_{0}^{z_{n}}\left(1-\hat{G}_{n}(x)\right) d x\right)^{2}}\right] .
$$

Notice that $\left((c-h) \int_{0}^{z_{n}} x \hat{g}_{n}(x) d x-p\left(1-\hat{G}_{n}\left(z_{n}\right)\right) E\left[X_{n}\right]\right)$ is strictly increasing in $z_{n}$, negative for small values of $z_{n}$, and positive for large values of $z_{n}$. Therefore, by the definition of $z_{n}^{T}$, we conclude that $r_{n}^{*}\left(z_{n}\right)$ is decreasing for $z_{n}<z_{n}^{T}$ and increasing for $z_{n}>z_{n}^{T}$.

Also from Equation (73) we derive

$$
\begin{aligned}
\frac{d^{2} r_{n}^{*}\left(z_{n}\right)}{d z_{n}^{2}}= & \frac{b}{b-1} \times \frac{1}{\left(\int_{0}^{z_{n}}\left(1-\hat{G}_{n}(x)\right) d x\right)^{3}} \\
& \times\left[\left((c-h) z_{n} \hat{g}_{n}\left(z_{n}\right)+p \hat{g}_{n}\left(z_{n}\right) E\left[X_{n}\right]\right) \int_{0}^{z_{n}}\left(1-\hat{G}_{n}(x)\right) d x\right. \\
& \left.-2\left(1-\hat{G}_{n}\left(z_{n}\right)\right)\left((c-h) \int_{0}^{z_{n}} x \hat{g}_{n}(x) d x-p\left(1-\hat{G}_{n}\left(z_{n}\right)\right) E\left[X_{n}\right]\right)\right],
\end{aligned}
$$

which implies the convexity of $r_{n}^{*}\left(z_{n}\right)$ for $z_{n}<z_{n}^{T}$.

From part (b) we get

$$
\frac{d y_{n}^{*}\left(z_{n}\right)}{d z_{n}}=a\left(r_{n}^{*}\left(z_{n}\right)\right)^{-b}\left[1-\frac{b z_{n}}{r_{n}^{*}\left(z_{n}\right)} \frac{d r_{n}^{*}\left(z_{n}\right)}{d z_{n}}\right]
$$

The stated results for $y_{n}^{*}\left(z_{n}\right)$ follow from Equation (75). 


\section{References}

[1] Agrawal, N., S. A. Smith. 1996. Estimating negative binomial demand for retail inventory management with unobservable lost sales. Naval Res. Logist. 43 839-861.

[2] Azoury, K. S. 1985. Bayes solution to dynamic inventory models under unknown demand distribution. Management Sci. 31(9) 1150-1160.

[3] Braden, D. J., M. Freimer. 1991. Informational dynamics of censored observations. Management Sci. 37(11) 1390-1404.

[4] Chen, L., E. L. Plambeck. 2005. Dynamic inventory management with learning about the demand distribution and substitution probability. Working paper, Stanford University.

[5] Dana, J. D., N. C. Petruzzi. 2001. Note: The newsvendor model with endogenous demand. Management Sci. 47(11) 1488-1497.

[6] Ding, X., M. L. Puterman, A. Bisi. 2002. The censored newsvendor and the optimal acquisition of information. Oper. Res. 50(3) 517-527.

[7] Harpaz, G., W. Y. Lee, R. L. Winkler. 1982. Learning, experimentation, and the optimal output decisions of a competitive firm. Management Sci. 28(6) 589-603.

[8] Iglehart, D. L. 1964. The dynamic inventory problem with unknown demand distribution. Management Sci. 10(3) 429-440.

[9] Karlin, S. 1960. Dynamic inventory policy with varying stochastic demands. Management Sci. 6(3) $231-258$.

[10] Lariviere, M. A., E. L. Porteus. 1999. Stalking information: Bayesian inventory management with unobserved lost sales. Management Sci. 45(3) 346-363. 
[11] Lovejoy, W. S. 1990. Myopic policies for some inventory models with uncertain demand distribution. Management Sci. 36(6) 724-738.

[12] Lovejoy, W. S. 1993. Suboptimal policies, with bounds, for parameter adaptive decision processes. Oper. Res. 41(3) 583-599.

[13] Lu, X., J. S. Song, K. Zhu. 2004. Dynamic inventory planning for perishable products with censored demand data. Working paper, Hong Kong University of Science and Technology and Duke University.

[14] Lu, X., J. S. Song, K. Zhu. 2005. Inventory control with unobservable lost sales and Bayesian updates. Working paper, Hong Kong University of Science and Technology and Duke University.

[15] Murray, G. R., E. A. Silver. 1966. A Bayesian analysis of the style goods inventory problem. Management Sci. 12(11) 785-797.

[16] Nahmias, S. 1994. Demand estimation in lost sales inventory systems. Naval Res. Logist. 41 739-757.

[17] Petruzzi, N. C., M. Dada. 1999. Pricing and the newsvendor problem: A review with extensions. Oper. Res. 47(2) 183-194.

[18] Petruzzi, N. C., M. Dada. 2001. Information and inventory recourse for a two-market, price-setting retailer. Manufacturing \& Service Operations Management 3 242-263.

[19] Petruzzi, N. C., M. Dada. 2002. Dynamic pricing and inventory control with learning. Naval Res. Logist. 49 303-325.

[20] Scarf, H. 1959. Bayes solutions of the statistical inventory problem. Ann. Math. Stat. 30 490-508.

[21] Scarf, H. 1960. Some remarks on Bayes solutions to the inventory problem. Naval Res. Logist. Quart. 7 591-596. 
[22] Silver, E. A., D. F. Pyke, R. Peterson. 1998. Inventory Management and Production Planning and Scheduling. John Wiley \& Sons, New York.

[23] Treharne, J. T., C. R. Sox. 2002. Adaptive inventory control for nonstationary demand and partial information. Management Sci. 48 (5) 607-624.

[24] Van Hee, K. M. 1978. Bayesian Control of Markov Chains. Mathematisch Centrum, Amsterdam, The Netherlands.

[25] Veinott, A., Jr. 1965. Optimal policy for a multi-product, dynamic non-stationary inventory problem. Management Sci. 12 206-222.

[26] Young, L. 1978. Price, inventory and the structure of uncertain demand. New Zealand Oper. Res. 6 157-177. 